\title{
MATHEMATICAL AND NUMERICAL ANALYSIS OF A MODEL FOR ANTI-ANGIOGENIC THERAPY IN METASTATIC CANCERS ${ }^{*, * *}$
}

\author{
SÉBASTIEN BENZEKRY ${ }^{1}$
}

\begin{abstract}
We introduce a phenomenological model for anti-angiogenic therapy in the treatment of metastatic cancers. It is a structured transport equation with a nonlocal boundary condition describing the evolution of the density of metastases that we analyze first at the continuous level. We present the numerical analysis of a lagrangian scheme based on the characteristics whose convergence establishes existence of solutions. Then we prove an error estimate and use the model to perform interesting simulations in view of clinical applications.
\end{abstract}

Mathematics Subject Classification. 35F16, 65M25, 92C50.

Received September 15, 2010. Revised May 3, 2011

Published online October 5, 2011.

\section{INTRODUCTION}

During the evolution of a cancer disease, a fundamental step for the tumour consists in provoking proliferation of the surrounding blood vessels and migration toward the tumour. This process, called tumoural neo-angiogenesis establishes a proper vascular network which ensures to the tumour supply of nutrients and allow the tumour to grow further than $2-3 \mathrm{~mm}$ diameter. It is also important in the metastatic process by making possible the spread of cancerous cells to the organism which then can develop in secondary tumours (metastases). Thus, an interesting therapeutic strategy first proposed by Folkman [16] in the seventies consists in blocking angiogenesis with the goal to starve the primary tumour by depriving it from nutrient supply. This can be achieved by inhibiting the action of the Vascular Endothelial Growth Factor molecule either with monoclonal antibodies or tyrosine kinase inhibitors. Although the concept of the therapy seems perfectly clear, the practical use of the anti-angiogenic (AA) drugs leaves various open questions regarding to the best temporal administration protocols. Indeed, AA treatments lead to relatively poor efficacy and can even provoke deleterious effects, especially on metastases [22]. Regarding to these therapeutic failures, it seems that the scheduling of the drug plays a major role. Indeed, as shown in the publication [15], different schedules for the same drug can lead to completely different results. Moreover, AA drugs are never given in a monotherapy but always combined with cytotoxic agents (also named chemotherapy) which act directly on the cancerous cells. Again, the scheduling of the drugs seems to be highly relevant [24] and the optimal combination schedule between these two types of drugs is still a clinical open question. Thus, the complex dynamics of tumoural growth and metastatic evolution have to be taken into account in the design of temporal administration protocols for anti-cancerous drugs.

Keywords and phrases. Anticancer therapy modelling, angiogenesis, structured population dynamics, lagrangian scheme.

* The author would like to express its gratitude to the following people for great support and helpful discussions: D. Barbolosi, A. Benabdallah, F. Hubert and F. Boyer. He expresses also his acknowledgments to anonymous referees for helpful comments and critics.

** This work was partially supported by the ANR project MEMOREX-PK.

${ }^{1}$ LATP, UMR 6632. Université de Provence, Technopole Château-Gombert, 39 rue F. Joliot Curie, 13453 Marseille Cedex 13, France.

Laboratoire de Toxicocinétique et Pharmacocinétique UMR-MD3, 27 boulevard Jean Moulin, 13005 Marseille, France.

benzekry@phare.normalesup.org 
In order to give answers to these questions, various mathematical models are being developed for tumoural growth including the angiogenic process. We can distinguish between two classes of models: mechanistic models (see for instance [9,21]) try to integrate the whole biology of the processes and comprise a large number of parameters; on the other hand phenomenological models aim at describing the tumoural growth without taking into account all the complexity levels (see [25] for a review and [6,13,17]). Most of these models deal only with growth of the primary tumour but in 2000, Iwata et al. [19] proposed a simple model for the evolution of the population of metastases, which was then further studied in $[2,11]$. This model did not include the angiogenic process in the tumoural growth and thus could not integrate a description of the effect of an AA drug. We combined it with the tumoural model introduced by Hahnfeldt et al. [17] which takes into account for angiogenesis. The resulting partial differential equation is part of the so-called structured population dynamics (see [23] for an introduction to the theory): it is a transport equation with a nonlocal boundary condition. Its mathematical analysis is not classical because the structuring variable is two-dimensional; as far as we know such two dimensional models have only been studied in the case where one structuring variable is the age and thus has constant velocity (see $[14,26]$ ). This is not the case in our situation and the theoretical analysis of the model without treatment (autonomous case) was performed in [7].

In this paper, we present some mathematical and numerical analysis of the model in the non-autonomous case that is, integrating both cytotoxic and AA treatments and with a general growth field $G$ (i.e. the growth law of the tumours) satisfying the hypothesis that there exists a positive constant $\delta$ such that $-G(t, \sigma) \cdot \nu(\sigma) \geq \delta>0$ for all $t>0$ and almost every $\sigma \in \partial \Omega$ where $\nu$ is the normal to the boundary $\partial \Omega$ of the domain $\Omega$ where metastases live, meaning that $G$ points inward the domain. We first simplify the problem by straightening the characteristics of the equation. We perform some theoretical analysis first at the continuous level (uniqueness and a priori estimates). Then we introduce an approximation scheme which follows the characteristics of the equation (lagrangian scheme). The introduction of such schemes in the area of size-structured population equations can be found in [1] for one-dimensional models. Here, we go further in the lagrangian approach by doing the change of variables straightening the characteristics and discretizing the simple resulting equation, in the case of a general class of two-dimensional non-autonomous models. We prove existence of the weak solution to the continuous problem through the convergence of this scheme via discrete a priori $L^{\infty}$ bounds and establish an error estimate in the case of more regular data.

Finally, we use this scheme to perform various simulations demonstrating the possible utility of the model. First, as a predictive tool for the number of metastases in order to refine the existing classifications of cancers regarding to metastatic aggressiveness. Secondly, the model can be used to test various temporal administration protocols of AA drugs in monotherapy or combined with a cytotoxic agent.

\section{MOdeL}

The model is based on the approach of $[2,11,19]$ to describe the evolution of a population of metastases represented by its density $\rho(t, X)$ with $X$ being the structuring variable, here two-dimensional $X=(x, \theta)$ with $x$ the size (=number of cells) and $\theta$ the so-called angiogenic capacity (maximal reachable size for a tumor, assumed to represent the degree of vascularization). It is a partial differential equation of transport type. The behavior of each individual of the population (metastasis), that is the growth rate $G(t, X)$ of each tumour is taken from [17] and is designed to take into account the angiogenic process, as well as the effect of both anti-angiogenic (AA) and cytotoxic drugs (CT). Its expression will be established in the following subsection. The model writes

$$
\begin{cases}\partial_{t} \rho(t, X)+\operatorname{div}(\rho(t, X) G(t, X))=0 & \forall(t, X) \in] 0, T[\times \Omega \\ -G \cdot \nu(t, \sigma) \rho(t, \sigma)=N(\sigma) \int_{\Omega} \beta(X) \rho(t, X) \mathrm{d} X+f(t, \sigma) & \forall(t, \sigma) \in] 0, T[\times \partial \Omega \\ \rho(0, X)=\rho^{0}(X) & \forall X \in \Omega,\end{cases}
$$

where $\Omega$, the birth rate $\beta(X)$, the repartition along the boundary $N(\sigma)$ and the source term $f(t, \sigma)$ will be specified in the sequel, $T$ is a positive time and $\nu$ is the unit external normal vector to the boundary $\partial \Omega$. 


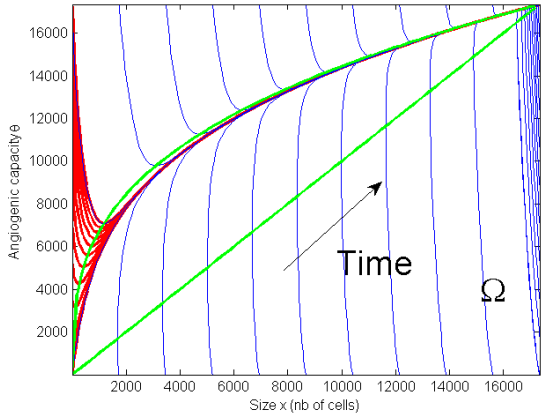

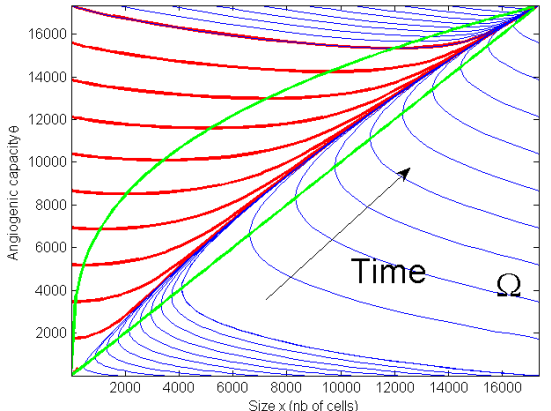

(B)

Figure 1. Two phase plans of the system (1.2)-(1.3), for different values of the parameters, without treatment $(h=e=0)$. In green, the nullclines. In both, $b:=\left(\frac{c}{d}\right)^{\frac{3}{2}}=17347$. (A) Parameters from [17]: $a=0.192, c=5.85, d=8.73 \times 10^{-3}$. (B) $a=0.192, c=0.1, d=$ $1.4923 \times 10^{-4}$

\subsection{The model of tumoural growth under angiogenic control (Hahnfeldt et al. [17]}

Let $x(t)$ denote the size (expressed in number of cells, homogeneous to a volume through the approximation $1 \mathrm{~mm}^{3} \simeq 10^{6}$ cells) of a given tumour at time $t$. The growth of the tumour is modeled by a gompertzian growth rate modified by a death term describing the action of a CT. Equation is:

$$
\frac{\mathrm{d} x}{\mathrm{~d} t}=g_{1}(t, x, \theta)=a x \ln \left(\frac{\theta}{x}\right)-h \gamma_{C}(t) H\left(x-x_{\min }\right),
$$

where $a$ is a parameter representing the velocity of the growth, $\theta$ the carrying capacity of the environment. This parameter is the maximal sustainable size by the environment and thus has size unit. The term $h \gamma_{C}(t) H\left(x-x_{\text {min }}\right)$ stands for the effect of a cytotoxic drug, where $\gamma_{C}$ is the concentration of the CT, $x_{\min }$ is a minimal size for the drug to be effective $\left(x_{\min } \geq 1\right)$ and the function $H$ is a regularization of the Heaviside function (for example $H(t)=1 / 2+1 / 2 \tanh (t / K)$, with $K$ being a parameter controlling the slope at 0 ), in order to avoid regularity issues in the analysis. The idea of [17] is to take $\theta$ not anymore as a parameter but as a variable of the time, representing the degree of vascularization of the tumour and called "angiogenic capacity". Indeed the tumoural growth is limited by the nutrient's supply provided by the surrounding vascularization and hence it makes sense to consider the carrying capacity of equation (1.2) as a representant of the degree of vascularization of the tumour. The variation rate for $\theta$ derived in [17] is:

$$
\frac{\mathrm{d} \theta}{\mathrm{d} t}=g_{2}(t, x, \theta)=c x-\mathrm{d} \theta x^{\frac{2}{3}}-e \gamma_{A}(t) H\left(\theta-\theta_{\min }\right)
$$

where the terms $c x$ and $-\mathrm{d} \theta x^{2 / 3}$ represent respectively the endogenous stimulation and inhibition of the vasculature and $e \gamma_{A}(t) H\left(\theta-\theta_{\min }\right)$ is the effect of an anti-angiogenic drug. The factor $2 / 3$ comes from the analysis of [17] which concluded that the ratio of the stimulation rate over the inhibition one should be homogeneous to the tumoural radius to the square. In Figure 1, we present some numerical simulations of the phase plan of the system (1.2), (1.3).

Following [17], we assume a one compartmental pharmacokinetic for the AA and do the same for the CT (in [17] there is no CT). We also assume that the drugs are administered as boli. This gives

$$
\gamma_{A}(t)=\sum_{i=1}^{N} D_{A} \mathrm{e}^{-c l r_{A}\left(t-t_{i}^{A}\right)} H\left(t-t_{i}^{A}\right)
$$


where the $t_{i}^{A}$ are the administration times of the AA, $D_{A}$ is the administered dose and $c l r_{A}$ the clearance. The expression for the $\mathrm{CT}$ is the same, with $\mathrm{C}$ instead of $\mathrm{A}$.

\subsection{Renewal equation for the density of metastasis}

We denote $X=(x, \theta)$ and $G(t, X)=\left(g_{1}(t, x, \theta), g_{2}(t, x, \theta)\right)$. Study of the behaviour of solutions to the system $(1.2)-(1.3)$ reveals convergence to an equilibrium point $(b, b)$ with $b=\left(\frac{c}{d}\right)^{\frac{3}{2}}$ when there is no treatment (see [12] for a study of this system without the CT term and the Figure 1 for a numerical illustration). This value $b$ hence appears as the maximal reachable size and angiogenic capacity for $(x, \theta)$ solving the system $(1.2),(1.3)$. We assume that each tumour has $x$ and $\theta$ satisfying $1 \leq x, \theta \leq b$ since they are both number of cells and thus cannot be less than 1 and cannot exceed $b$ from the previous argument. Defining $\Omega=[1, b] \times[1, b]$ this means that we consider each tumour as a particle evolving in $\Omega$ with the velocity $G$. Writing a balance law for the density $\rho(t, X)$ we have

$$
\left.\partial_{t} \rho+\operatorname{div}(\rho G)=0, \quad \forall(t, X) \in\right] 0, T[\times \Omega
$$

that we endow with an initial condition $\rho^{0} \in L^{\infty}(\Omega)$.

Metastases do not only grow in size and angiogenic capacity, they are also able to emit new metastases. We denote by $\mathbf{b}(\sigma, x, \theta)$ the birth rate of new metastasis with size and angiogenic capacity $\sigma \in \partial \Omega$ by metastasis of size $x$ and angiogenic capacity $\theta$, and by $f(t, \sigma)$ the term corresponding to metastasis produced by the primary tumour. Expressing the equality between the number of metastasis arriving in $\Omega$ per unit time (l.h.s in the following equality, since $G(t, \sigma) \cdot \nu(\sigma)<0, \forall t>0$, a.e. $\sigma \in \partial \Omega$ from expressions (1.2)-(1.3), using the fact that the treatment is ineffective for tumors with size or angiogenic capacity equal to 1) and the total rate of new metastasis created by both the primary tumour and metastasis themselves (r.h.s.), we should have for all $t>0$

$$
-\int_{\partial \Omega} \rho(t, \sigma) G(t, \sigma) \cdot \nu(\sigma) \mathrm{d} \sigma=\int_{\partial \Omega} \int_{\Omega} \mathbf{b}(\sigma, X) \rho(t, X) \mathrm{d} X+f(t, \sigma) \mathrm{d} \sigma .
$$

We assume that the emission rate of the primary and secondary tumours are equal and thus take $f(t, \sigma)=$ $\mathbf{b}\left(\sigma, X_{p}(t)\right)$ where $X_{p}(t)$ represents the primary tumour and solves the ODE system (1.2)-(1.3). An important feature of the model is to assume that the vasculature of the neo-metastasis is independent from the one which emitted it. This means that there exists a function $N: \partial \Omega \rightarrow \mathbb{R}$ and a function $\beta: \Omega \rightarrow \mathbb{R}$ such that $\mathbf{b}(\sigma, X)=N(\sigma) \beta(x, \theta)$. The function $\beta$ is the emission rate of new metastasis per tumour per unit time and $N$ is their distribution at birth.

We assume that the newly created metastases have size $x=1$, in view of the following remarks: (a) the passing vascular holes by which a metastasis pass to escape from the tumor have diameter of order $100 \mathrm{~nm}$. It is hard to imagine that more than one cell (whose typical size is the micrometer) could pass through such a small hole. (b) If the cells detach from the tumor, it means that the cadherin (transmembrane proteins responsible for cell-cell adhesion) rate falls. Thus it seems unlikely that the cells lose cadherins from one side and keep sufficient to form a cluster on the other side. (c) Even in the assumption of the detachment of a cluster of cells, it would be composed of at most a dozen of cells since (a) and the hypothesis of size 1 cell for the neo-metastasis would stay in a convenient approximation. This implies that the support of $N$ is included in $\{\sigma \in \partial \Omega ; \sigma=(1, \theta), 1 \leq \theta \leq b\}$. Although we have no reference to provide about the shape of the angiogenic birth distribution of the metastases, we assume it to be uniformly centered around a mean value $\theta_{0}$, thus we take

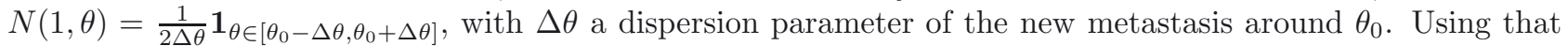
equation (1.5) should be verified for each subset of the boundary, we obtain the boundary condition of (1.1).

For the numerical simulations, in order to reduce the computational cost, we examine replacing the function $N(\sigma)$ by a Dirac measure. This means doing the assumption that all the metastases are born with the same angiogenic capacity $\theta_{0}$. In [8], we demonstrate that if we take $N^{\varepsilon}(1, \theta)=\frac{1}{2 \varepsilon} \mathbf{1}_{\theta \in\left[\theta_{0}-\varepsilon, \theta_{0}+\varepsilon\right]}$ and let $\varepsilon$ go to zero the solution of the problem (1.1), in the case of an autonomous velocity field $G$ and initial condition equal to zero, converges to the measure solution of a limit problem consisting in replacing $N$ by a Dirac measure in $\left(1, \theta_{0}\right)$. 
Following the modeling of [19] for the colonization rate $\beta$ we take

$$
\beta(x, \theta)=m x^{\alpha}
$$

with $m$ the colonization coefficient and $\alpha$ the so-called fractal dimension of blood vessels infiltrating the tumour. The parameter $\alpha$ expresses the geometrical distribution of the vessels in the tumour. For example, if the vasculature is superficial then $\alpha$ is assigned to $2 / 3$ thus making $x^{\alpha}$ proportional to the area of the surface of the tumour (assumed to be spheroidal). Else if the tumour is homogeneously vascularised, then $\alpha$ is supposed to be equal to 1 .

\section{Analysis at the continuous level}

In the autonomous case, that is when $G$ depends only on $X$ and there is no treatment, the analysis of equation (1.1) has been performed in [7]. It was proven existence, uniqueness, regularity and asymptotic behavior of solutions. We present now some analysis in equation (1.1) with a more general growth field $G$ than the one defined in Section 1.2.

Let $\Omega=] 1, b\left[^{2}\right.$ and $G: \mathbb{R} \times \bar{\Omega} \rightarrow \mathbb{R}^{2}$ be a $\mathcal{C}^{1}$ vector field. We make the following assumption on $G$ :

$$
\exists \delta>0,-G(t, \sigma) \cdot \nu(\sigma) \geq \delta>0 \quad \forall 0 \leq t \leq T, \text { a.e. } \sigma \in \partial \Omega
$$

which means that $G$ points inward all along the boundary for all times and that the metastases can't go out of $\Omega$ (and thus are never removed from the system). We do the following assumptions on the data:

$$
\rho^{0} \in L^{\infty}(\Omega), \beta \in L^{\infty}(\Omega), N \in L^{\infty}(\partial \Omega), N \geq 0, \int_{\partial \Omega} N(\sigma) \mathrm{d} \sigma=1, f \in L^{\infty}(] 0, T[\times \partial \Omega) .
$$

\section{Remark 2.1.}

- In the case of $G$ being the one of Section 1.2 if there is no treatment (that is, if $e=h=0$, or $t \leq t_{1}$ ) then we don't have $-G(t, \sigma) \cdot \nu(\sigma) \geq \delta>0$ all along the boundary since $G$ vanishes at the point $(b, b)$. But since the problem was solved in this case (see [7]) we consider that the time 0 is the starting time of the treatment and that either $e$ or $h$ is positive, which makes the assumption (2.1) true. Notice that this assumption implies that the treatment is ineffective on metastases with minimal size or angiogenic capacity, which is true in our case (see the expressions (1.2)-(1.3)).

- The following analysis at the continuous level extends to the case where $\Omega$ has a boundary which is piecewise $\mathcal{C}^{1}$ except in a finite number of points.

Definition 2.1 (weak solution). We say that $\rho \in L^{\infty}(] 0, T[\times \Omega)$ is a weak solution of the problem (1.1) if for all test function $\phi$ in $\mathcal{C}^{1}([0, T] \times \bar{\Omega})$ with $\phi(T, \cdot)=0$

$$
\begin{aligned}
\int_{0}^{T} \int_{\Omega} \rho(t, X)\left[\partial_{t} \phi(t, X)+G(t, X) \cdot \nabla \phi(t, X)\right] \mathrm{d} X \mathrm{~d} t & +\int_{\Omega} \rho^{0}(X) \phi(0, X) \mathrm{d} X \\
& +\int_{0}^{T} \int_{\partial \Omega}\{N(\sigma) B(t, \rho)+f(t, \sigma)\} \phi(t, \sigma)=0
\end{aligned}
$$

where we denoted $B(t, \rho):=\int_{\Omega} \beta(X) \rho(t, X) \mathrm{d} X$.

Remark 2.2. By approximating a Lipschitz function by $\mathcal{C}^{1}$ ones, it is possible to prove that the definition of weak solutions would be equivalent with test functions in $W^{1, \infty}([0, T] \times \bar{\Omega})$ vanishing at time $T$. 


\subsection{Change of variables}

Our approach is based on the method of characteristics and consists in straightening the characteristics to simplify the problem through a change of variables. Let $\Phi(t ; \tau, \sigma)$ be the solution of the differential equation

$$
\left\{\begin{array}{c}
\frac{\mathrm{d}}{\mathrm{d} t} \Phi(t ; \tau, \sigma)=G(t, \Phi(t ; \tau, \sigma)) \\
\Phi(\tau ; \tau, \sigma)=\sigma
\end{array} .\right.
$$

For each time $t>0$, we define the entrance time $\tau^{t}(X)$ and entrance point $\sigma^{t}(X)$ for a point $X \in \Omega$ :

$$
\tau^{t}(X):=\inf \{0 \leq \tau \leq t ; \Phi(\tau ; t, X) \in \Omega\}, \sigma^{t}(X):=\Phi\left(\tau^{t}(X) ; t, X\right) .
$$

We consider the sets

$$
\Omega_{1}^{t}=\left\{X \in \Omega ; \tau^{t}(X)>0\right\}, \Omega_{2}^{t}=\left\{X \in \Omega ; \tau^{t}(X)=0\right\}
$$

and

$$
Q_{1}:=\left\{(t, X) \in[0, T] \times \bar{\Omega} ; X \in \bar{\Omega}_{1}^{t}\right\}, Q_{2}:=\left\{(t, X) \in[0, T] \times \bar{\Omega} ; X \in \bar{\Omega}_{2}^{t}\right\} .
$$

We also define $\widetilde{Q_{1}}:=\{(t, \tau, \sigma) ; 0 \leq \tau \leq t \leq T, \sigma \in \partial \Omega\}=\Phi^{-1}\left(Q_{1}\right)$ and notice that

$\Sigma_{1}:=[0, T] \times \partial \Omega=\left\{(t, X) ; \tau^{t}(X)=0\right\}$, and $\Sigma_{2}=\{(t, \Phi(t ; 0, \sigma)) ; 0 \leq t \leq T, \sigma \in \partial \Omega\}=\left\{(t, X) ; \tau^{t}(X)=0\right\}$

See Figure 2 for an illustration. We can now introduce the changes of variables that we will constantly use in the sequel.

Proposition 2.1 (change of variables). The maps

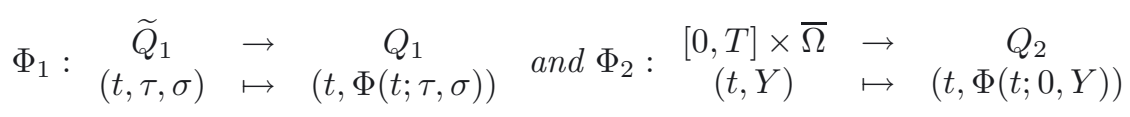

are bilipschitz. The inverse of $\Phi_{1}$ is $(t, X) \mapsto\left(t, \tau^{t}(X), \sigma^{t}(X)\right)$ and the inverse of $\Phi_{2}$ is $(t, X) \mapsto(t, Y(X))$ with $Y(X)=\Phi(0 ; t, X)$. Denoting $J_{1}(t ; \tau, \sigma)=\left|\operatorname{det}\left(D \Phi_{1}\right)\right|$ and $J_{2}(t ; Y)=\left|\operatorname{det}\left(D \Phi_{2}\right)\right|$, where D $\Phi_{i}$ stands for the differential of $\Phi_{i}$, we have:

$$
J_{1}(t ; \tau, \sigma)=|G(\tau, \sigma) \cdot \vec{\nu}(\sigma)| \mathrm{e}^{\int_{\tau}^{t} \operatorname{div} G(u, \Phi(u ; \tau, \sigma)) \mathrm{d} u} \text { and } J_{2}(t ; Y)=\mathrm{e}^{\int_{0}^{t} \operatorname{div} G(u, \Phi(u ; 0, Y)) \mathrm{d} u} .
$$

We refer to the appendix for the proof of this result and to Figure 2 for an illustration.

Using these changes of variables we can write for a function $f \in L^{1}(] 0, T[\times \Omega)$

$$
\int_{0}^{T} \int_{\Omega} f(X) \mathrm{d} X=\int_{0}^{T} \int_{0}^{t} \int_{\partial \Omega} f\left(\Phi_{1}(t ; \tau, \sigma)\right) J_{1}(t ; \tau, \sigma) \mathrm{d} \sigma \mathrm{d} \tau+\int_{0}^{T} \int_{\Omega} f\left(\Phi_{2}(t ; 0, Y)\right) J_{2}(t ; Y) \mathrm{d} Y .
$$

We want to decompose equation (1.1) into two subequations: one for the contribution of the boundary term and one for the contribution of the initial condition. Defining

$$
\widetilde{\rho}_{1}(t, \tau, \sigma):=\rho(t, \Phi(t ; \tau, \sigma)) J_{1}(t ; \tau, \sigma) \text { and } \widetilde{\rho}_{2}(t, y):=\rho(t, \Phi(t ; 0, Y)) J_{2}(t ; Y)
$$

we have, when the solution is regular: $\partial_{t} \widetilde{\rho}_{1}=\left(\partial_{t} \rho+\operatorname{div}(\rho G)\right) J_{1}=0$ and the same for $\widetilde{\rho}_{2}$. It is thus natural to introduce the following equations

$$
\begin{cases}\partial_{t} \widetilde{\rho}_{1}(t, \tau, \sigma)=0 & 0<\tau \leq t<T, \sigma \in \partial \Omega \\ \widetilde{\rho}_{1}(\tau, \tau, \sigma)=N(\sigma) \widetilde{B}\left(\tau, \widetilde{\rho}_{1}, \widetilde{\rho}_{2}\right)+f(\tau, \sigma) & 0<\tau<T, \sigma \in \partial \Omega\end{cases}
$$




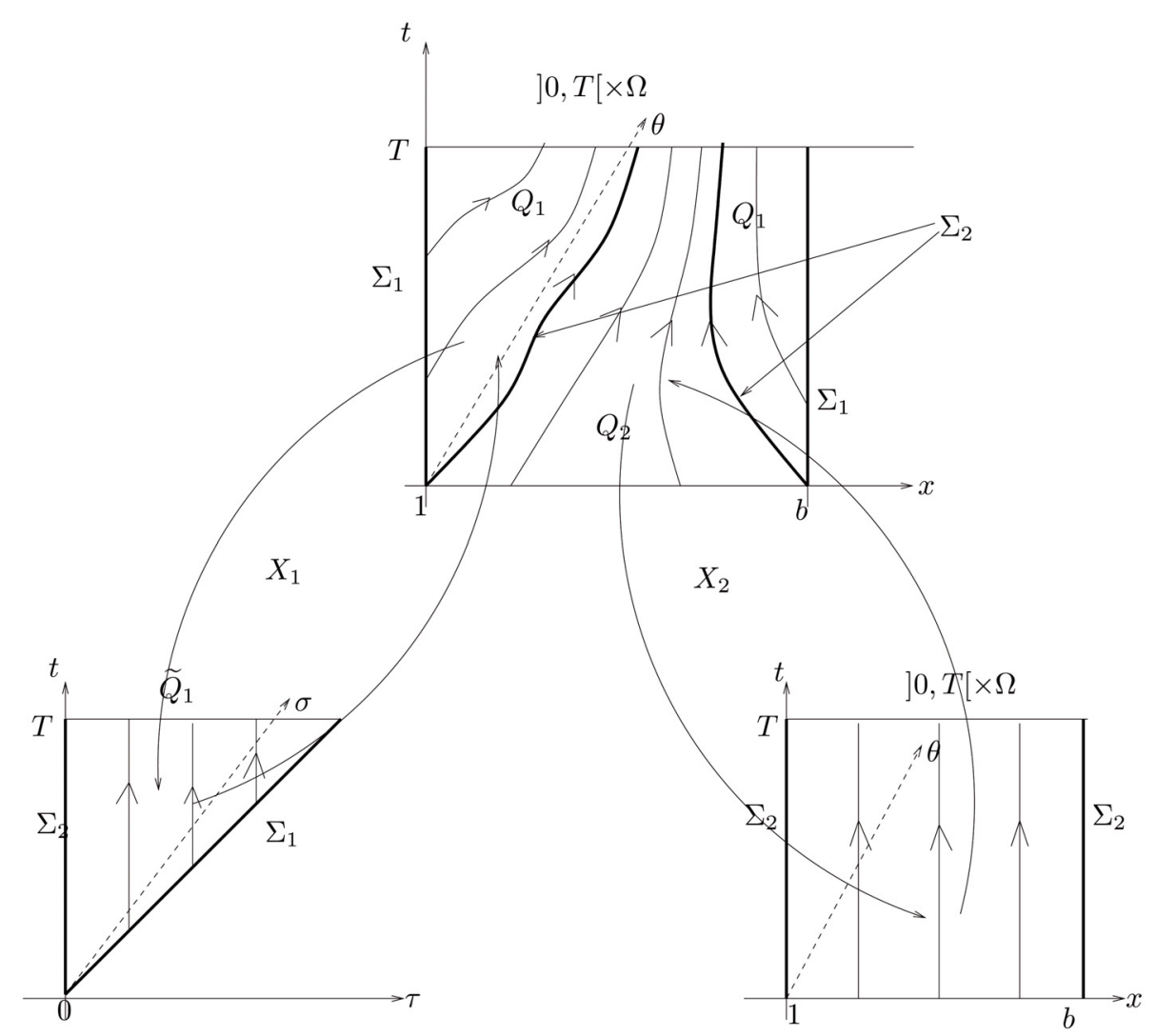

Figure 2. The two changes of variables $\Phi_{1}$ and $\Phi_{2}$ (represented only on the plane $\theta=1$ ).

where we denoted

$$
\widetilde{B}\left(\tau, \widetilde{\rho}_{1}, \widetilde{\rho}_{2}\right)=\int_{0}^{\tau} \int_{\partial \Omega} \beta(\Phi(\tau ; s, \sigma)) \widetilde{\rho}_{1}(\tau, s, \sigma) \mathrm{d} \sigma \mathrm{d} s+\int_{\Omega} \beta(\Phi(\tau ; 0, Y)) \widetilde{\rho}_{2}(\tau, Y) \mathrm{d} Y,
$$

and

$$
\begin{cases}\partial_{t} \widetilde{\rho}_{2}=0 & t>0, Y \in \Omega \\ \widetilde{\rho}_{2}(0 ; Y)=\rho^{0}(Y) & Y \in \Omega .\end{cases}
$$

We precise the definition of weak solutions to these equations.

Definition 2.2. We say that a couple $\left(\widetilde{\rho}_{1}, \widetilde{\rho}_{2}\right) \in L^{\infty}\left(\widetilde{Q}_{1}\right) \times L^{\infty}(] 0, T[\times \Omega)$ is a weak solution of equations $(2.7)-(2.8)$ if for all $\widetilde{\phi}_{1} \in \mathcal{C}^{1}\left(\widetilde{Q}_{1}\right)$ with $\widetilde{\phi}_{1}(T, \cdot)=0$ we have:

$$
\int_{0}^{T} \int_{0}^{t} \int_{\partial \Omega} \widetilde{\rho}_{1}(t, \tau, \sigma) \partial_{t} \widetilde{\phi}_{1}(t, \tau, \sigma) \mathrm{d} \sigma \mathrm{d} \tau \mathrm{d} t+\int_{0}^{T} \int_{\partial \Omega}\left\{N(\sigma) \widetilde{B}\left(t, \widetilde{\rho}_{1}, \widetilde{\rho}_{2}\right)+f(t, \sigma)\right\} \widetilde{\phi}_{1}(t, t, \sigma)=0
$$

and for all $\widetilde{\phi}_{2} \in \mathcal{C}^{1}([0, T] \times \bar{\Omega})$ with $\widetilde{\phi}_{2}(T, \cdot)=0$ we have

$$
\int_{0}^{T} \int_{\Omega} \widetilde{\rho}_{2}(t, Y) \partial_{t} \widetilde{\phi}_{2}(t, Y) \mathrm{d} t+\int_{\Omega} \rho^{0}(Y) \widetilde{\phi}_{2}(0, Y) \mathrm{d} Y=0
$$


Remark 2.3. If $\widetilde{\rho}_{1}$ is a regular function which solves (2.7), then the weak formulation is satisfied since we have:

$$
\begin{aligned}
\int_{0}^{T} \int_{0}^{t} \int_{\partial \Omega} \widetilde{\rho}_{1}(t, \tau, \sigma) \partial_{t} \widetilde{\phi}_{1}(t, \tau, \sigma) \mathrm{d} \sigma \mathrm{d} \tau \mathrm{d} t=\underbrace{\int_{0}^{T} \int_{\partial \Omega} \widetilde{\phi}_{1}(T, \tau, \sigma) \widetilde{\rho}_{1}(T, \tau, \sigma) \mathrm{d} \tau \mathrm{d} \sigma}_{=0} \\
-\int_{0}^{T} \int_{0}^{t} \widetilde{\phi}_{1}(t, \tau, \sigma) \partial_{t} \widetilde{\rho}_{1}(t, \tau, \sigma) \mathrm{d} \sigma \mathrm{d} \tau \mathrm{d} t-\int_{0}^{T} \int_{\partial \Omega} \widetilde{\phi}_{1}(t, t, \sigma) \widetilde{\rho}_{1}(t, t, \sigma) \mathrm{d} \sigma \mathrm{d} t .
\end{aligned}
$$

We prove now the following theorem, establishing the equivalence between the problem (1.1) and the problem (2.7)-(2.8).

Theorem 2.1 (equivalence between problem (1.1) and problem (2.7)-(2.8)). Let $\rho \in L^{\infty}(] 0, T[\times \Omega)$ be a weak solution of equation (1.1). Then $\left(\widetilde{\rho}_{1}, \widetilde{\rho}_{2}\right)$ given by (2.6) is a weak solution of (2.7)-(2.8). Conversely, if $\widetilde{\rho}_{1}$ and $\widetilde{\rho}_{2}$ are weak solutions of (2.7) and (2.8), then the function defined by

$$
\rho(t, X):=\widetilde{\rho}_{1}\left(t, \tau^{t}(X), \sigma^{t}(X)\right) J_{1}^{-1}\left(t, \tau^{t}(X), \sigma^{t}(X)\right) \mathbf{1}_{X \in \Omega_{1}^{t}}+\widetilde{\rho}_{2}(t, Y(X)) J_{2}^{-1}(t, Y(X)) \mathbf{1}_{X \in \Omega_{2}^{t}}
$$

is a weak solution of (1.1).

Proof.

- Direct implication. Let $\rho$ be a weak solution of the equation (1.1). We will prove that $\widetilde{\rho}_{2}$ defined by (2.6) solves (2.8). Let $\widetilde{\phi}_{2} \in \mathcal{C}^{1}([0, T] \times \bar{\Omega})$ with $\widetilde{\phi}_{2}(T, \cdot)=0$. We define, for $X \in Q_{2}, \phi_{2}(t, X):=\widetilde{\phi}_{2}(t, Y(X)) \in$ $W^{1, \infty}\left(Q_{2}\right)$ and we intend to extend it in a Lipschitz function of $[0, T] \times \bar{\Omega}$ so that we can use it as a test function in the weak formulation for $\rho$ (see Rem. 2.2). We define, for $(t, \tau, \sigma) \in \widetilde{Q}_{1}, \widetilde{\phi}_{1}^{\varepsilon}(t, \tau, \sigma)=\widetilde{\phi}_{2}(t, \sigma) \zeta_{\varepsilon}(\tau)$ with $\zeta_{\varepsilon}(\tau)$ being a truncature function in $\mathcal{C}^{1}\left(\left[0,+\infty[)\right.\right.$ such that $0 \leq \zeta_{\varepsilon} \leq 1, \zeta_{\varepsilon}(0)=1, \zeta_{\varepsilon}(\tau)=0$ for $\tau \geq \varepsilon$. Then $\widetilde{\phi}_{1}^{\varepsilon} \in W^{1, \infty}\left(\widetilde{Q}_{1}\right)$ and we set $\phi_{1}^{\varepsilon}(t, X):=\widetilde{\phi}_{1}^{\varepsilon}\left(t, \tau^{t}(X), \sigma^{t}(X)\right) \in W^{1, \infty}\left(Q_{1}\right)$ since $\tau^{t}(X)$ and $\sigma^{t}(X)$ are Lipschitz from Proposition 2.1. We define then

$$
\phi^{\varepsilon}:=\left\{\begin{array}{ll}
\phi_{1}^{\varepsilon} \text { on } Q_{1} \\
\phi_{2} \text { on } Q_{2}
\end{array} .\right.
$$

The function $\phi^{\varepsilon}$ is Lipschitz on $Q_{1}$, Lipschitz on $Q_{2}$ and $\phi^{\varepsilon} \in \mathcal{C}([0, T] \times \bar{\Omega})$ since $Q_{1} \cap Q_{2}=\left\{(t, X) ; \tau^{t}(X)=0\right\}$.

Thus $\phi^{\varepsilon} \in W^{1, \infty}([0, T] \times \bar{\Omega})$ with $\phi^{\varepsilon}(T, \cdot)=0$. Using $\phi^{\varepsilon}$ as a test function in $(2.3)$, we have

$$
\begin{gathered}
\int_{Q_{1}} \rho\left[\partial_{t} \phi_{1}^{\varepsilon}+G \cdot \nabla \phi_{1}^{\varepsilon}\right] \mathrm{d} X \mathrm{~d} t+\int_{0}^{T} \int_{\partial \Omega}\{N(\sigma) B(t, \rho)+f(t, \sigma)\} \phi_{1}^{\varepsilon}(t, \sigma) \mathrm{d} t \mathrm{~d} \sigma \\
+\int_{Q_{2}} \rho\left[\partial_{t} \phi_{2}+G \cdot \nabla \phi_{2}\right] \mathrm{d} X \mathrm{~d} t+\int_{\Omega} \rho^{0}(X) \phi_{2}(0, X) \mathrm{d} X=0=I_{\varepsilon}^{1}+I_{2} .
\end{gathered}
$$

By doing the change of variables $\Phi_{1}$ in the term $I_{1}^{\varepsilon}$ and noticing that $\phi_{1}^{\varepsilon}(t, \sigma)=\widetilde{\phi}_{1}^{\varepsilon}(t, t, \sigma)=\widetilde{\phi}_{2}(t, \sigma) \zeta_{\varepsilon}(t)$, we obtain

$$
I_{1}^{\varepsilon}=\int_{0}^{T} \int_{0}^{t} \widetilde{\rho}_{1}(t, \tau, \sigma) \partial_{t} \widetilde{\phi}_{1}(t, \sigma) \zeta^{\varepsilon}(\tau) \mathrm{d} \sigma \mathrm{d} \tau \mathrm{d} t+\int_{0}^{T} \int_{\partial \Omega} B(t, \rho) \widetilde{\phi}_{2}(t, \sigma) \zeta^{\varepsilon}(t) \mathrm{d} \sigma \underset{\varepsilon \rightarrow 0}{\longrightarrow} 0 .
$$

Now doing the change of variables $\Phi_{2}$ in the second term $I_{2}$ and noticing that $\partial_{t} \widetilde{\phi}_{2}(t, Y)=\partial_{t}\left(\phi_{2}(t, \Phi(t ; 0, Y))\right)=$ $\partial_{t} \phi_{2}(t, \Phi(t ; 0, Y))+G(t, \Phi(t ; 0, Y)) \cdot \nabla \phi_{2}(t, \Phi(t ; 0, Y))$ gives the result. The equation on $\widetilde{\rho}_{1}$ is proved in the same way.

- Reverse implication. Let $\widetilde{\rho}_{1}$ and $\widetilde{\rho}_{2}$ be solutions of (2.7) and (2.8) respectively. Define $\rho(t, X)$ by (2.11), and consider a test function $\phi \in \mathcal{C}^{1}([0, T] \times \bar{\Omega})$ with $\phi(T, \cdot)=0$. Then $\phi_{1}:=\phi_{\mid Q_{1}} \in \mathcal{C}^{1}\left(Q_{1}\right)$, with $\phi_{1}(T, \cdot)=0$, thus $\widetilde{\phi}_{1}(t, \tau, \sigma):=\phi_{1}\left(t, \Phi_{1}(\tau, \sigma)\right)$ is valid as a test function in the weak formulation of $(2.7)$. In the same way 
$\widetilde{\phi}_{2}(t, Y):=\phi_{2}\left(t, \Phi_{2}(Y)\right)$ with $\phi_{2}:=\phi_{\mid Q_{2}}$ is valid as a test function for (2.8). Thus we have

$$
\begin{gathered}
\int_{\widetilde{Q}_{1}} \widetilde{\rho}_{1}(t, \tau, \sigma) \partial_{t} \widetilde{\phi}_{1}(t, \tau, \sigma) \mathrm{d} \sigma \mathrm{d} \tau \mathrm{d} t+\int_{0}^{T} \int_{\partial \Omega} \widetilde{B}\left(t, \widetilde{\rho}_{1}, \widetilde{\rho}_{2}\right) \widetilde{\phi}_{1}(t, t, \sigma) \mathrm{d} \sigma \mathrm{d} t \\
\quad+\int_{0}^{T} \int_{\Omega} \widetilde{\rho}_{2}(t, y) \partial_{t} \widetilde{\phi}_{2}(t, y) \mathrm{d} t \mathrm{~d} Y+\int_{\Omega} \rho^{0}(y) \widetilde{\phi}_{2}(0, y) \mathrm{d} Y=0 .
\end{gathered}
$$

Doing the changes of variables gives the weak formulation of (1.1).

This theorem simplifies the structure of the problem (1.1). In some sense, it formalizes the method of characteristics in the framework of weak solutions for our problem. The characteristics are straightened (see Fig. 2) and the directional derivative along the field $(t, G)$ is transformed in only a time derivative. Moreover, integrating the jacobians (which contains the transformation of areas) in the definitions of $\widetilde{\rho}_{1}$ and $\widetilde{\rho}_{2}$, these functions are constant in time. The continuous analysis and discrete approximation of the problem (1.1) is thus simplified.

\subsection{A priori continuous estimates and uniqueness}

To obtain a priori estimates on the solutions of equation in order to prove uniqueness, we need a trace for a solution to the transport equation as well as the fact that $|\rho|$ also solves the equation. These issues are treated in the papers of Bardos [4] and Beals and Protopopescu [5]. We will use a result well adapted for our aim from Boyer [10].

Theorem 3.1 from [10] . Let $\rho \in L^{\infty}(] 0, T[\times \Omega)$ be a solution, in the distribution sense, to the equation:

$$
\partial_{t} \rho+\operatorname{div}(\rho G)=0 .
$$

(i) The function $\rho$ lies in $\mathcal{C}\left([0, T] ; L^{p}(\Omega)\right)$, for any $1 \leq p<\infty$. Furthermore, $\rho$ is continuous in time with values in $L^{\infty}(\Omega)$ weak-*.

(ii) There exists a function $\gamma \rho \in L^{\infty}(] 0, T\left[\times \partial \Omega ;\left|\mathrm{d} \mu_{G}\right|\right)$, with $\mathrm{d} \mu_{G}=(G \cdot \nu) \mathrm{d} t \mathrm{~d} \sigma$, such that for any $h \in \mathcal{C}^{1}(\mathbb{R})$, for any test function $\phi \in \mathcal{C}^{1}([0, T] \times \bar{\Omega})$, and for any $\left[t_{0}, t_{1}\right] \subset[0, T]$, we have

$$
\begin{aligned}
\int_{t_{0}}^{t_{1}} \int_{\Omega} h(\rho)\left(\partial_{t} \phi\right. & +\operatorname{div}(G \phi)) \mathrm{d} t \mathrm{~d} X+\int_{\Omega} h\left(\rho\left(t_{0}\right)\right) \phi\left(t_{0}\right) \mathrm{d} X-\int_{\Omega} h\left(\rho\left(t_{1}\right)\right) \phi\left(t_{1}\right) \mathrm{d} X \\
& -\int_{t_{0}}^{t_{1}} \int_{\partial \Omega} h(\gamma \rho) \phi G \cdot \nu \mathrm{d} t \mathrm{~d} \sigma-\int_{t_{0}}^{t_{1}} \int_{\Omega} h^{\prime}(\rho) \rho \operatorname{div}(G) \phi \mathrm{d} t \mathrm{~d} X=0 .
\end{aligned}
$$

\section{Remark 2.4.}

- By approximating the function $s \mapsto|s|$ by $\mathcal{C}^{1}$ functions, it is possible to show that the formula (2.13) stands with $h(s)=|s|$.

- The second point of the proposition implies in particular that $h(\rho)$ has a trace which is $h(\gamma \rho)$.

- In [10], this proposition is proved in the case of a much less regular field $G$ but with the technical assumption that $\operatorname{div}(G)=0$, which is not the case here. Though, the proof can be extended to our case.

Thanks to this result, we can prove the following proposition.

Proposition 2.2 (continuous a priori estimates). Let $\rho \in L^{\infty}(] 0, T[\times \Omega)$ be a weak solution of equation (1.1). The following estimates hold

$$
\|\rho(t, \cdot)\|_{L^{1}(\Omega)} \leq \mathrm{e}^{t|| \beta \|_{L^{\infty}}}\left\|\rho^{0}\right\|_{L^{1}(\Omega)}+\int_{0}^{t} \mathrm{e}^{(t-s)\|\beta\|_{L^{\infty}}} \int_{\partial \Omega}|f(s, \sigma)| \mathrm{d} \sigma \mathrm{d} s
$$


and

$$
\|\rho\|_{L^{\infty}(] 0, T[\times \Omega)} \leq C_{\infty}
$$

with

$$
C_{\infty}=\left(\|N\|_{L^{\infty}}\|\beta\|_{L^{\infty}}\|\rho\|_{L^{\infty}\left(L^{1}\right)}+\|f\|_{L^{\infty}}\right)\|G\|_{L^{\infty}} \mathrm{e}^{T\|\operatorname{div} G\|_{L^{\infty}}}+\left\|\rho^{0}\right\|_{L^{\infty}} \mathrm{e}^{T\|\operatorname{div} G\|_{L^{\infty}}}
$$

Proof.

- Estimate in $L^{1}$. Let $\rho$ be a weak solution of equation (1.1). Then in particular it solves (2.12) in the sense of distributions. Thus Proposition 2.2 applies and gives a trace $\gamma \rho \in L^{\infty}(] 0, T\left[\times \partial \Omega ;\left|d_{\mu G}\right|\right)$. Now, by using (2.13) with $h(s)=s$ and the definition of weak solutions to equation (1.1) we have that for all $\phi \in \mathcal{C}_{c}^{1}([0, T[\times \bar{\Omega})$

$$
\int_{0}^{T} \int_{\partial \Omega} \gamma \rho(t, \sigma) \phi(t, \sigma) G(t, \sigma) \cdot \nu \mathrm{d} \sigma \mathrm{d} t=\int_{0}^{T} \int_{\partial \Omega}\left\{N(\sigma) \int_{\Omega} \beta(X) \rho(t, X) \mathrm{d} X+f(t, \sigma)\right\} \phi(t, \sigma) \mathrm{d} \sigma \mathrm{d} t
$$

which gives

$$
-\gamma \rho(t, \sigma) G(t, \sigma) \cdot \nu=N(\sigma) \int_{\Omega} \beta(X) \rho(t, X) \mathrm{d} X+f(t, \sigma), \quad \text { a.e. }
$$

In view of Remark 2.4, we know that $|\rho|$ is also a weak solution to equation (1.1), with initial data $\left|\rho^{0}\right|$ and boundary data $|N(\sigma) B(t, \rho)+f(t, \sigma)|$. By integrating this equation on $\Omega$ and using the divergence formula, we obtain in the distribution sense:

$$
\frac{\mathrm{d}}{\mathrm{d} t} \int_{\Omega}|\rho(t, X)| \mathrm{d} X=-\int_{\partial \Omega} G(t, \sigma) \cdot \nu|\gamma \rho(t, \sigma)| \mathrm{d} \sigma=\int_{\partial \Omega}|N(\sigma) B(t, \rho)+f(t, \sigma)| \mathrm{d} \sigma
$$

and thus

$$
\frac{\mathrm{d}}{\mathrm{d} t} \int_{\Omega}|\rho(t, X)| \mathrm{d} X \leq\|\beta\|_{\infty} \int_{\Omega}|\rho(t, X)| \mathrm{d} X+|f(t, \sigma)|
$$

A Gronwall lemma concludes.

- Estimate in $L^{\infty}$. Using the Proposition 2.1, we have $\widetilde{\rho}_{1}$ and $\widetilde{\rho}_{2}$ solving (2.7) and (2.8). By doing the changes of variables, using the definitions of $\widetilde{\rho}_{1}$ and $\widetilde{\rho}_{2}$ and the formulas $(2.5)$, we see that

$$
\begin{gathered}
\|\rho(t, \cdot)\|_{L^{1}(\Omega)}=\left\|\widetilde{\rho}_{1}(t, \cdot)\right\|_{L^{1}(] 0, t[\times \partial \Omega)}+\left\|\widetilde{\rho}_{2}(t, \cdot)\right\|_{L^{1}(\Omega)}, \quad \forall t>0 \\
\|\rho\|_{L^{\infty}(] 0, T[\times \Omega)} \leq\left\|\widetilde{\rho}_{1}\right\|_{L^{\infty}\left(\widetilde{Q}_{1}\right)}\|G\|_{L^{\infty}(\partial \Omega)} \mathrm{e}^{T\|\operatorname{div} G\|_{\infty}}+\left\|\widetilde{\rho}_{2}\right\|_{L^{\infty}(] 0, T[\times \Omega)} \mathrm{e}^{T\|\operatorname{div} G\|_{\infty}} .
\end{gathered}
$$

But solving explicitely equation (2.7), we have

$$
\begin{aligned}
\left|\widetilde{\rho}_{1}(t, \tau, \sigma)\right| & =\left|\widetilde{\rho}_{1}(\tau, \tau, \sigma)\right|=\left|N(\sigma) \widetilde{B}\left(t, \widetilde{\rho}_{1}, \widetilde{\rho}_{2}\right)+f(t, \sigma)\right| \\
& \leq\|N\|_{\infty}\|\beta\|_{\infty}\left(\left\|\widetilde{\rho}_{1}(\tau, \cdot)\right\|_{L^{1}}+\left\|\widetilde{\rho}_{2}(\tau, \cdot)\right\|_{L^{1}}\right)+\|f\|_{L^{\infty}} \\
& \leq\left.\|N\|_{\infty}\|\beta\|_{\infty}\|\rho(\tau, \cdot)\|\right|_{L^{1}}+\|f\|_{L^{\infty}} .
\end{aligned}
$$

On the other hand, for $\widetilde{\rho}_{2}$ we have $\left\|\widetilde{\rho}_{2}\right\|_{L^{\infty}(] 0, T[\times \Omega)}=\left\|\widetilde{\rho}_{2}(0)\right\|_{L^{\infty}(\Omega)}=\left\|\rho^{0}\right\|_{L^{\infty}(\Omega)}$.

Remark 2.5. The expression (2.16) shows that in the case of a zero boundary data $f$, the trace $\gamma \rho$ has some extra regularity, namely it is $\mathcal{C}\left([0, T] ; L^{1}(\partial \Omega)\right)$. 
Corollary 2.1 (uniqueness). If $\rho$ and $\rho^{\prime}$ are two weak solutions of the problem (1.1), then $\rho=\rho^{\prime}$ almost everywhere.

\section{Approximated solutions}

As can be seen in Figure 1, for the parameters taken from the literature, the area where the solution is positive (characteristics coming from a part of the left edge of the square, represented in red) is very small compared to the area of the domain. A finite differences or finite volume scheme written on a cartesian mesh of the square would not exploit this feature of the model and would loose a lot of time calculating the solution in areas where it is zero. Therefore, we rather use a lagrangian scheme based on discretizing the characteristics of equation which in our framework consists in discretizing equations (2.7)-(2.8), in view of Proposition 2.1.

In this section, we build a weak solution to equation (1.1). We will achieve the existence by convergence of an approximation scheme to the problem (2.7)-(2.8) where the difficulty is restricted to the approximation of the boundary condition. Then we establish an error estimate in the case of more regular data. In order to avoid heavy notations, we forget about the tilda when referring to the problem (2.7)-(2.8).

\subsection{Construction of approximated solutions of the problem (2.7)-(2.8)}

Let $0=t_{0}<\ldots<t_{k}<\ldots<t_{K+1}=T$ be a uniform subdivision of $[0, T]$ with $t_{k+1}-t_{k}=\delta t$. For the equation (2.8), let the uniform subdivisions $1=x_{1}<\ldots<x_{l}<\ldots<x_{L+1}=b$ and $1=\theta_{1}<\ldots<\theta_{m}<\ldots<$ $\theta_{L+1}=b$, with $x_{l+1}-x_{l}=\theta_{m+1}-\theta_{m}=\delta x$. The scheme for equation (2.8) is then given by:

$$
\left\{\begin{array}{ll}
\rho_{2}^{0}(l, m)=\frac{1}{(\delta x)^{2}} \int_{x_{l}}^{x_{l+1}} \int_{\theta_{m}}^{\theta_{m+1}} \rho^{0}(x, \theta) \mathrm{d} x \mathrm{~d} \theta & 1 \leq l, m \leq L \\
\rho_{2}^{k+1}(l, m)=\rho_{2}^{k}(l, m) & 0 \leq k \leq K, 1 \leq l, m \leq L
\end{array} .\right.
$$

That is, $\rho_{2}^{k}(l, m)=\rho_{2}^{0}(l, m)$ for all $k, l, m$.

For the discretization of equation (2.7), for each $k$ let $0=\tau_{0}<\ldots<\tau_{i}<\ldots<\tau_{k}=t_{k}$ with $\tau_{i+1}-\tau_{i}=\delta t$. Let $\sigma:[0,4 b] \rightarrow \partial \Omega$ be defined by $\sigma(s)=\left\{\begin{array}{ll}(1,1+s) & s \in[0, b] \\ (1+s-b, b) & s \in[b, 2 b] \\ (b, 3 b-s) & s \in[2 b, 3 b] \\ (4 b-s, b) & s \in[3 b, 4 b]\end{array}\right.$ be a parametrization of $\partial \Omega$ with $\left|\sigma^{\prime}(s)\right|=1$ a.e., so that for $g \in L^{1}(\partial \Omega)$ we have $\int_{\partial \Omega} g(\sigma) \mathrm{d} \sigma=\int_{0}^{4 b} g(\sigma(s)) \mathrm{d} s$. Let $0=s_{1}<\ldots<s_{j}<\ldots<s_{M+1}=4 b$ be an uniform subdivision with $s_{j+1}-s_{j}=\delta \sigma$. The scheme is given by

$$
\begin{cases}\rho_{1}^{0}(0, j)=N_{j} B^{0}\left(\left(\rho_{2}^{0}\right)_{l, m}\right)+f_{j}^{0} & 1 \leq j \leq M \\ \rho_{1}^{k+1}(i, j)=\rho_{1}^{k}(i, j) & 0 \leq k \leq K, 0 \leq i \leq k, 1 \leq j \leq M \\ \rho_{1}^{k+1}(k+1, j)=N_{j} B^{k+1}\left(\rho_{1}^{k+1}, \rho_{2}^{k+1}\right)+f_{j}^{k+1} & 0 \leq k \leq K, 1 \leq j \leq M\end{cases}
$$

with

$$
B^{k}\left(\rho_{1}^{k}, \rho_{2}^{k}\right)=\sum_{i=1}^{k-1} \sum_{j=1}^{M} \beta_{i, j}^{1} \rho_{1}^{k}(i, j) \delta t \delta \sigma+\sum_{l, m=1}^{L} \beta_{l, m}^{2} \rho_{2}^{k}(l, m)(\delta x)^{2}
$$

meant to approximate

$$
\int_{0}^{t_{k}} \int_{\partial \Omega} \beta\left(\Phi\left(t_{k} ; \tau, \sigma\right)\right) \rho_{1}\left(t_{k}, \tau, \sigma\right) \mathrm{d} \tau \mathrm{d} \sigma+\int_{\Omega} \beta\left(\Phi\left(t_{k} ; 0, Y\right)\right) \rho_{2}\left(t_{k}, Y\right) \mathrm{d} Y
$$




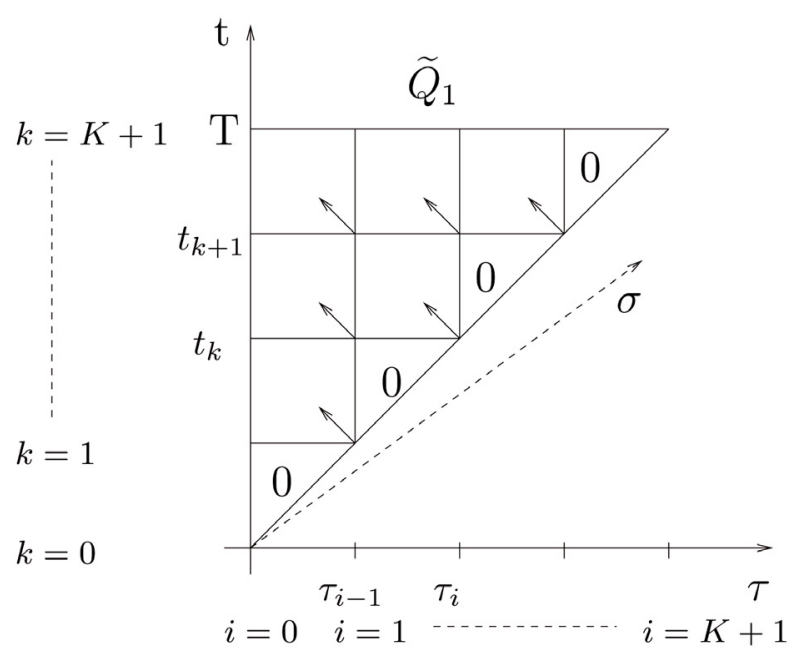

FIGURE 3. Description of the discretization grid for $\widetilde{Q}_{1}$, only in the $(\tau, t)$ plane. The arrows indicate the index used in assigning values to $\rho_{1, h}$ in each mesh (formula (3.4)).

and

$$
\begin{aligned}
\beta_{i, j}^{1}: & =\frac{1}{\delta t \delta \sigma} \int_{\tau_{i}}^{\tau_{i+1}} \int_{\sigma_{j}}^{\sigma_{j+1}} \beta\left(\Phi\left(t_{k} ; \tau, \sigma\right)\right) \mathrm{d} \sigma \mathrm{d} \tau, \beta_{l, m}^{2}:=\int_{x_{l}}^{x_{l+1}} \int_{\theta_{m}}^{\theta_{m+1}} \beta\left(\Phi\left(t_{k} ; 0,(x, \theta)\right)\right) \mathrm{d} x \mathrm{~d} \theta \\
f_{j}^{k}: & =\frac{1}{\delta t \delta \sigma} \int_{t_{k}}^{t_{k+1}} \int_{\sigma_{j}}^{\sigma_{j+1}} f(t, \sigma) \mathrm{d} \sigma \mathrm{d} t, N_{j}:=\frac{1}{\delta \sigma} \int_{\sigma_{j}}^{\sigma_{j+1}} N(\sigma) \mathrm{d} \sigma .
\end{aligned}
$$

Notice that the schemes (3.1) and (3.2) are well-posed since the definition of $\rho_{1}^{k+1}(k+1, j)$ involves values of $\rho_{1}^{k+1}(i, j)$ only with $0 \leq i \leq k$. We denote by $h=\delta t+\delta \sigma+\delta x$ and define now piecewise constant functions $\rho_{1, h}$ and $\rho_{2, h}$ on $\widetilde{Q}_{1}$ and $[0, T[\times \bar{\Omega}$ by, for $0 \leq k \leq K, 1 \leq i \leq k, 1 \leq j \leq M$ and $1 \leq l, m \leq L$

$$
\begin{array}{ll}
\rho_{1, h}(t, \tau, \sigma(s))=\rho_{1}^{k}(i, j) & \text { for } t \in\left[t_{k}, t_{k+1}[, \tau \in] \tau_{i-1}, \tau_{i}\right], s \in\left[s_{j}, s_{j+1}[\right. \\
\rho_{1, h}(t, \tau, \sigma(s))=0 & \text { for } t \in\left[t_{k}, t_{k+1}[, \tau \in] t_{k}, t\right], s \in\left[s_{j}, s_{j+1}[\right. \\
\rho_{2, h}(t, x, \theta)=\rho_{2}^{k}(l, m) & \text { for } t \in\left[t_{k}, t_{k+1}\left[, x \in\left[x_{l}, x_{l+1}\left[, \theta \in\left[\theta_{m}, \theta_{m+1}[.\right.\right.\right.\right.\right.
\end{array}
$$

See Figure 3 for an illustration. Notice that we have

$$
\left\|\rho_{1, h}\left(t_{k}, \cdot\right)\right\|_{L^{1}(] 0, t_{k}[\times \partial \Omega)}=\sum_{i=1}^{k} \sum_{j=1}^{M}\left|\rho_{1}^{k}(i, j)\right| \delta t \delta \sigma,\left\|\rho_{2, h}\left(t_{k}, \cdot\right)\right\|_{L^{1}(\Omega)}=\sum_{l, m=1}^{M}\left|\rho_{2}^{k}(l, m)\right|(\delta x)^{2} .
$$

\section{Remark 3.1.}

- We take the same discretization step in $x$ and $\theta$ for $\rho_{2}$ but it would work the same with two different steps.

- For more regular data, we could take point values instead of (3.3).

- It will be clear from the following that the scheme would converge the same regardless to the value that we give to $\rho_{1}^{0}(0, j)$. 


\subsection{Discrete a priori estimates}

We prove the equivalent of Proposition 2.2 in the discrete case. Notice that there exists a constant $C_{\sigma}$ such that $\sum_{j=1}^{M} N_{j} \delta \sigma \leq \int_{\partial \Omega} N(\sigma) \mathrm{d} \sigma+C_{\sigma} \delta \sigma=1+C_{\sigma} \delta \sigma:=\|N\|_{h}$ and $\sum_{j=1}^{M} f_{j}^{k+1} \delta \sigma \leq\|f\|_{L^{\infty}(] 0, T\left[; L^{1}(\partial \Omega)\right)}+C_{\sigma} \delta \sigma:=$ $\|f\|_{h}$.

Proposition 3.1 (discrete a priori estimates). Let $\left(\rho_{1}^{k}(i, j)\right)_{k, i, j}$ and $\left(\rho_{2}^{k}(l, m)\right)_{k, l, m}$ be given by (3.1) and (3.2) respectively. Then for all $k$

$$
\begin{aligned}
& \left\|\rho_{2, h}\left(t_{k}, \cdot\right)\right\|_{L^{1}(\Omega)}=\left\|\rho^{0}\right\|_{L^{1}(\Omega)}, \quad\left\|\rho_{2, h}\right\|_{L^{\infty}(j 0, T[\times \Omega)}=\left\|\rho^{0}\right\|_{L^{\infty}(\Omega)} \\
& \left\|\rho_{1, h}\left(t_{k}, \cdot\right)\right\|_{L^{1}(] 0, t_{k}[\times \partial \Omega)} \leq \mathrm{e}^{t_{k}\|\beta\|_{L^{\infty}}\|N\|_{h}}\left\{\left\|\rho^{0}\right\|_{L^{1}(\Omega)}+\frac{\|f\|_{h}}{\|\beta\|_{L^{\infty}}\|N\|_{h}}\right\}, \\
& \left\|\rho_{1, h}\right\|_{L^{\infty}\left(\widetilde{Q}_{1}\right)} \leq\|N\|_{L^{\infty}}\|\beta\|_{L^{\infty} \max _{k}}\left(\left\|\rho_{1, h}\left(t_{k}, \cdot\right)\right\|_{L^{1}}+\left\|\rho^{0}\right\|_{L^{1}}\right)+\|f\|_{L^{\infty}} .
\end{aligned}
$$

Moreover, if $\rho^{0} \geq 0$ then $\rho_{1}^{k}(i, j), \rho_{2}^{k}(l, m) \geq 0$ for all $k, i, j, l, m$.

Proof. The non-negativity of the scheme is straightforward from the definition. The estimate for $\rho_{2, h}$ follows directly from the scheme (3.2). For the $L^{1}$ estimate on $\rho_{1, h}$ we compute, using the scheme (3.1)

$$
\begin{aligned}
\left\|\rho_{1, h}\left(t_{k+1}, \cdot\right)\right\|_{L^{1}(] 0, t_{k+1}[\times \partial \Omega)} & =\sum_{i=1}^{k+1} \sum_{j=1}^{M}\left|\rho^{k+1}(i, j)\right| \delta t \delta \sigma \\
& =\sum_{i=1}^{k} \sum_{j=1}^{M}\left|\rho^{k}(i, j)\right| \delta t \delta \sigma+\left|B^{k+1}\left(\rho_{1}^{k+1}, \rho_{2}^{k+1}\right)\right| \delta t \sum_{j=1}^{M} N_{j} \delta \sigma+\delta t \sum_{j=1}^{M}\left|f_{j}^{k+1}\right| \delta \sigma \\
& \leq\left\|\rho_{1, h}\left(t_{k}\right)\right\|_{L^{1}(] 0, t_{k}[\times \partial \Omega)}+\left|B^{k+1}\left(\rho_{1}^{k+1}, \rho_{2}^{k+1}\right)\right| \delta t|| N\left\|_{h}+\delta t\right\| f \|_{h} .
\end{aligned}
$$

Now from the expression of $B^{k+1}\left(\rho_{1}^{k+1}, \rho_{2}^{k+1}\right)$

$$
\left|B^{k+1}\left(\rho_{1}^{k+1}, \rho_{2}^{k+1}\right)\right| \leq\|\beta\|_{L^{\infty}}\left\|\rho_{1, h}\left(t_{k}, \cdot\right)\right\|_{L^{1}}+\|\beta\|_{L^{\infty}}\left\|\rho_{2, h}\left(t_{k}, \cdot\right)\right\|_{L^{1}}
$$

Thus we obtain

$$
\left\|\rho_{1, h}\left(t_{k+1}, \cdot\right)\right\|_{L^{1}} \leq\left(1+\|\beta\|_{L^{\infty}} \delta t\|N\|_{h}\right)\left\|\rho_{1, h}\left(t_{k}, \cdot\right)\right\|_{L^{1}}+\|\beta\|_{L^{\infty}} \delta t|| N\left|\left\|_{h}\right\| \rho_{2, h}\left(t_{k}, \cdot\right)\left\|_{L^{1}}+\delta t|| f\right\|_{h}\right.
$$

Now using a discrete Gronwall Lemma we obtain

$$
\left.\left\|\rho_{1, h}\left(t_{k+1}, \cdot\right)\right\|\right|_{L^{1}} \leq \mathrm{e}^{\|\beta\|_{L^{\infty}}\|N\|_{h} t_{k}}\left\{\left\|\rho_{1, h}\left(t_{0}, \cdot\right)\right\|_{L^{1}}+\frac{\|\beta\|_{L^{\infty}}\|N\|\left\|_{h}\right\| \rho_{2, h}\left(t_{k}, \cdot\right)\left\|_{L^{1}}+\right\| f \|_{h}}{\|\beta\|_{L^{\infty}}\|N\|_{h}}\right\} .
$$

Using $\left\|\rho_{1, h}\left(t_{0}, \cdot\right)\right\|_{L^{1}}=0$ and $\left\|\rho_{2, h}\left(t_{k}, \cdot\right)\right\|_{L^{1}(\Omega)}=\left\|\rho^{0}\right\|_{L^{1}(\Omega)}$ ends the proof of the $L^{1}$ estimate. 
For the $L^{\infty}$ estimate, we remark that

$$
\begin{aligned}
\left\|\rho_{1, h}\right\|_{L^{\infty}\left(\widetilde{Q}_{1}\right)} & =\max _{k} \max _{i, j}\left|\rho_{1}^{k}(i, j)\right|=\max _{k} \max _{j}\left(\left|B^{k}\left(\rho_{1}^{k}, \rho_{2}^{k}\right) N_{j}+f_{j}^{k}\right|\right) \leq\|N\|_{L^{\infty}} \max _{k}\left|B^{k}\left(\rho_{1}^{k}, \rho_{2}^{k}\right)\right|+\|f\|_{L^{\infty}} \\
& \leq\|N\|_{L^{\infty}}\|\beta\|_{L^{\infty}} \max _{k}\left(\left.\left\|\rho_{1, h}\left(t_{k}, \cdot\right)\right\|\right|_{L^{1}}+\left\|\rho_{2, h}\left(t_{k}, \cdot\right)\right\|_{L^{1}}\right)+\|f\|_{L^{\infty}} .
\end{aligned}
$$

\subsection{Application to existence of solutions to the continuous problem $(2.7)-(2.8)$}

Theorem 3.1 (existence). Under the assumptions $(2.2)$, there exists $\rho_{1} \in L^{\infty}\left(\widetilde{Q}_{1}\right)$ and $\rho_{2} \in L^{\infty}(] 0, T[\times \Omega)$ such that $\rho_{1, h} \underset{h \rightarrow 0}{\longrightarrow} \rho_{1}$ and $\rho_{2, h} \underset{h \rightarrow 0}{\longrightarrow} \rho_{2}$ for the weak-* topology of $L^{\infty}$. Furthermore, $\left(\rho_{1}, \rho_{2}\right)$ is the unique weak solution of $(2.7)-(2.8)$.

Proof. Uniqueness of the solution is straightforward for the problem (2.8) and for the problem (2.7) it follows from the $L^{1}$ estimate on $\rho_{1}$ which can be derived following the proof of Proposition 2.2. The proof for the existence is rather classical and consists in passing to the limit in discrete weak formulations of (2.7) and (2.8). From the previous proposition, we obtain that the families $\left\{\rho_{1, h}\right\}_{\delta t, \delta \sigma}$ and $\left\{\rho_{2, h}\right\}_{\delta t, \delta x}$ are bounded in $L^{\infty}$ and thus there exist $\rho_{1} \in L^{\infty}\left(\widetilde{Q}_{1}\right), \rho_{2} \in L^{\infty}(] 0, T[\times \Omega)$ and some subsequences $\rho_{1, h_{n}}$ and $\rho_{2, h_{n}}$ such that $\rho_{1, h_{n}} \underset{h_{n} \rightarrow 0}{\longrightarrow} \rho_{1}$ and $\rho_{2, h_{n}} \underset{h_{n} \rightarrow 0}{\rightarrow} \rho_{2}$ for the weak-* topology of $L^{\infty}$. We have to prove now that $\left(\rho_{1}, \rho_{2}\right)$ is a weak solution of (2.7)-(2.8). The uniqueness of solutions to equation implies then by a standard argument that the whole sequence converges. It remains to prove that $\left(\rho_{1}, \rho_{2}\right)$ solves $(2.7)-(2.8)$.

- The function $\rho_{2}$ is a weak solution of (2.8). Let $\phi_{2}$ be a test function for (2.8). We have

$$
\begin{aligned}
\int_{0}^{T} \int_{\Omega} \rho_{2, h_{n}}(t, Y) \partial_{t} \phi_{2}(t, Y) \mathrm{d} Y \mathrm{~d} t & =\sum_{k=0}^{K} \sum_{l, m=1}^{L} \rho_{2}^{k}(l, m) \int_{t_{k}}^{t_{k+1}} \int_{x_{l}}^{x_{l+1}} \int_{\theta_{m}}^{\theta_{m+1}} \partial_{t} \phi_{2}(t, x, \theta) \mathrm{d} \theta \mathrm{d} x \mathrm{~d} t \\
& =\sum_{k=0}^{K} \sum_{l, m=1}^{L} \rho_{2}^{k}(l, m) \Phi_{2}\left(t_{k+1}, l, m\right)(\delta x)^{2}-\sum_{k=0}^{K} \sum_{l, m=1}^{L} \rho_{2}^{k}(l, m) \Phi_{2}\left(t_{k}, l, m\right)(\delta x)^{2}
\end{aligned}
$$

where we denoted $\Phi_{2}\left(t_{k}, l, m\right):=\frac{1}{(\delta x)^{2}} \int_{x_{l}}^{x_{l+1}} \int_{\theta_{m}}^{\theta_{m+1}} \phi_{2}\left(t_{k}, x, \theta\right) \mathrm{d} \theta \mathrm{d} x$. Using the scheme $\left(\rho_{2}^{k}(l, m)\right.$ is constant in $k$ ) and $\Phi_{2}\left(t_{K+1}, l, m\right)=0$ since $t_{K+1}=T$, we obtain

$$
\begin{gathered}
\int_{0}^{T} \int_{\Omega} \rho_{2, h_{n}}(t, Y) \partial_{t} \phi_{2}(t, Y) \mathrm{d} Y \mathrm{~d} t=\sum_{l, m=1}^{L} \rho_{2}^{K}(l, m) \Phi_{2}(T, l, m)(\delta x)^{2}-\sum_{l, m=1}^{L} \rho_{2}^{0}(l, m) \Phi_{2}(0, l, m)(\delta x)^{2} \\
=-\sum_{l, m=1}^{L} \rho_{2}^{0}(l, m) \Phi_{2}(0, l, m)(\delta x)^{2}=-\int_{\Omega} \rho_{2, h_{n}}^{0}(Y) \phi(0, Y) \underset{h_{n} \rightarrow 0}{\longrightarrow}-\int_{\Omega} \rho^{0}(Y) \phi(0, Y) \mathrm{d} Y
\end{gathered}
$$

since $\rho_{2, h_{n}}^{0} \underset{h_{n} \rightarrow 0}{\stackrel{L^{1}}{\longrightarrow}} \rho^{0}$. Observing that the left hand side converges to $\int_{0}^{T} \int_{\Omega} \rho_{2} \partial_{t} \phi_{2}(t, Y) \mathrm{d} Y \mathrm{~d} t$ gives the result.

- The function $\rho_{1}$ is a weak solution of (2.7). Let $\phi_{1}$ be a test function for (2.7). Then the same calculation as above shows, with $\Phi_{1}\left(t_{k}, i, j\right):=\frac{1}{\delta t \delta \sigma} \int_{\tau_{i-1}}^{\tau_{i}} \int_{\sigma_{j}}^{\sigma_{j+1}} \phi_{1}\left(t_{k}, \tau, \sigma\right) \mathrm{d} \sigma \mathrm{d} \tau$ and using that $\Phi_{1}\left(t_{K+1}, i, j\right)=0$ 
as well as $\rho_{1}^{k+1}(i, j)=\rho_{1}^{k}(i, j)$ for $1 \leq i \leq k$ and $1 \leq j \leq M$

$$
\begin{aligned}
\int_{\widetilde{Q}_{1}} \rho_{1, h_{n}}(t, \tau, \sigma) \partial_{t} \phi_{1}(t, \tau, \sigma) \mathrm{d} \sigma \mathrm{d} \tau \mathrm{d} t= & \sum_{k=1}^{K} \sum_{i=1}^{k} \sum_{j=1}^{M} \rho_{1}^{k}(i, j) \Phi_{1}\left(t_{k+1}, i, j\right) \delta t \delta \sigma-\sum_{k=1}^{K} \sum_{i=1}^{k} \sum_{j=1}^{M} \rho_{1}^{k}(i, j) \Phi_{1}\left(t_{k}, i, j\right) \delta t \delta \sigma \\
= & \sum_{i=1}^{K} \sum_{j=1}^{M} \rho_{1}^{K}(i, j) \Phi_{1}\left(t_{K+1}, i, j\right) \delta t \delta \sigma+\sum_{k=1}^{K-1} \sum_{i=1}^{k} \sum_{j=1}^{M} \rho_{1}^{k}(i, j) \Phi_{1}\left(t_{k+1}, i, j\right) \delta t \delta \sigma \\
& -\sum_{k=1}^{K-1} \sum_{i=1}^{k+1} \sum_{j=1}^{M} \rho_{1}^{k+1}(i, j) \Phi_{1}\left(t_{k+1}, i, j\right) \delta t \delta \sigma-\sum_{j=1}^{M} \rho_{1}^{1}(1, j) \Phi_{1}\left(t_{1}, 1, j\right) \delta t \delta \sigma \\
= & -\sum_{k=1}^{K-1} \sum_{j=1}^{M} \rho_{1}^{k+1}(k+1, j) \Phi_{1}\left(t_{k+1}, k+1, j\right) \delta t \delta \sigma-\sum_{j=1}^{M} \rho_{1}^{1}(1, j) \Phi_{1}\left(t_{1}, 1, j\right) \delta t \delta \sigma \\
= & -\sum_{k=1}^{K} \sum_{j=1}^{M}\left(N_{j} B^{k}\left(\rho_{1}^{k}, \rho_{2}^{k}\right)+f_{j}^{k}\right) \Phi_{1}\left(t_{k}, k, j\right) \delta t \delta \sigma
\end{aligned}
$$

Defining the following piecewise constant functions: $B_{h}\left(t, \rho_{1, h}, \rho_{2, h}\right)=B^{k}\left(\rho_{1}^{k}, \rho_{2}^{k}\right), N_{h}(\sigma(s))=N_{j}, f_{h}(t, \sigma(s))=$ $f_{j}^{k}$ and $\Phi_{1, h}(t, \sigma(s))=\Phi_{1}\left(t_{k}, k, j\right)$ on $\left[t_{k}, t_{k+1}\left[\times\left[s_{j}, s_{j+1}[\right.\right.\right.$, the previous equality reads

$$
\int_{\widetilde{Q}_{1}} \rho_{1, h_{n}}(t, \tau, \sigma) \partial_{t} \phi_{1}(t, \tau, \sigma) \mathrm{d} \sigma \mathrm{d} \tau \mathrm{d} t=\int_{\delta t}^{T} \int_{\partial \Omega}\left(B_{h_{n}}\left(t, \rho_{1, h_{n}}, \rho_{2, h_{n}}\right) N_{h_{n}}(\sigma)+f_{h_{n}}(t, \sigma)\right) \Phi_{1, h_{n}}(t, \sigma) \mathrm{d} \sigma \mathrm{d} t
$$

We need the following lemma in order to conclude.

Lemma 3.1. We have

$$
B_{h_{n}}\left(t, \rho_{1, h_{n}}, \rho_{2, h_{n}}\right) \underset{h_{n} \rightarrow 0}{\rightarrow} \widetilde{B}\left(t, \rho_{1}, \rho_{2}\right) *-L^{\infty}(] 0, T[) .
$$

Proof. We define the piecewise constant function $\beta_{h}^{1}(\tau, \sigma)$ as for $N_{h}$ and $f_{h}$ and $\beta_{h}^{2}(X)=\beta_{l, m}^{2}$ for $X \in$ $\left[x_{l}, x_{l+1}\left[\times\left[\theta_{m}, \theta_{m+1}\left[\right.\right.\right.\right.$. Let $t \in\left[t_{k}, t_{k+1}[\right.$, then

$$
B_{h}\left(t, \rho_{1, h}, \rho_{2, h}\right)=B^{k}\left(\rho_{1}^{k}, \rho_{2}^{k}\right)=\int_{0}^{t} \int_{\partial \Omega} \beta_{h}^{1}(\tau, \sigma) \rho_{1, h}(t, \tau, \sigma) \mathrm{d} \tau \mathrm{d} \sigma-\sum_{j=1}^{M} \beta_{k, j}^{1} \rho_{1}^{k}(k, j) \delta t \delta \sigma+\sum_{l, m=1}^{L} \beta_{l, m}^{2} \rho_{2}^{k}(l, m)(\delta x)^{2}
$$

since we defined $\rho_{h}(t, \tau, \sigma)=0$ for $\left.\left.\tau \in\right] t_{k}, t\right]$. Thus, for $\psi \in L^{1}(] 0, T[)$ we have

$$
\begin{aligned}
\int_{0}^{T} B_{h}\left(t, \rho_{1, h}, \rho_{2, h}\right) \psi(t) \mathrm{d} t & =\int_{0}^{T} \int_{0}^{t} \int_{\partial \Omega} \beta_{h}^{1}(\tau, \sigma) \rho_{1, h}(t, \tau, \sigma) \psi(t) \mathrm{d} \sigma \mathrm{d} \tau \mathrm{d} t-\delta t \sum_{k=0}^{K} \sum_{j=1}^{M} \beta_{k, j}^{1} \rho_{1}^{k}(k, j) \int_{t_{k}}^{t_{k+1}} \psi(t) \mathrm{d} t \delta \sigma \\
& +\int_{0}^{T} \int_{\Omega} \beta_{h}^{2}(X) \rho_{2, h}(t, X) \psi(t) \mathrm{d} X \mathrm{~d} t
\end{aligned}
$$

and we obtain the result by using $\rho_{1, h_{n}} \underset{h_{n} \rightarrow 0}{\rightarrow} \rho_{1} *-L^{\infty}, \rho_{2, h_{n}} \underset{h_{n} \rightarrow 0}{\rightarrow} \rho_{2} *-L^{\infty}, \beta_{h_{n}} \underset{h_{n} \rightarrow 0}{\stackrel{L^{1}}{\longrightarrow}} \beta,\left\|\beta_{h_{n}}\right\|_{L^{\infty}} \leq C$ and noticing that the second term goes to zero in view of the $L^{\infty}$ bounds on $\rho_{1, h}$ (Prop. 3.1) and $\beta$. 
Using the lemma as well as $N_{h_{n}}, f_{h_{n}} \underset{h_{n} \rightarrow 0}{\rightarrow} N, f *-L^{\infty},\left\|N_{h_{n}}\right\|_{L^{\infty}} \leq C$ and $\Phi_{1, h_{n}} \frac{\mathcal{C}([0, T] \times \partial \Omega)}{h_{n} \rightarrow 0} \phi(t, t, \sigma)$, the previous calculations give

$$
\int_{\widetilde{Q}_{1}} \rho_{1, h_{n}}(t, \tau, \sigma) \partial_{t} \phi_{1}(t, \tau, \sigma) \mathrm{d} \sigma \mathrm{d} \tau \mathrm{d} t \underset{h \rightarrow 0}{\longrightarrow}-\int_{0}^{T} \int_{\partial \Omega}\left\{N(\sigma) \widetilde{B}\left(t, \rho_{1}, \rho_{2}\right)+f(t, \sigma)\right\} \phi(t, t, \sigma) \mathrm{d} \sigma \mathrm{d} t .
$$

On the other hand the left hand side also goes to $\int_{\widetilde{Q}_{1}} \widetilde{\rho}_{1}(t, \tau, \sigma) \partial_{t} \phi_{1}(t, \tau, \sigma) \mathrm{d} \sigma \mathrm{d} \tau \mathrm{d} t$. This proves that $\rho_{1}$ verifies Definition 2.2 and ends the proof.

\subsection{Error estimate for the problem (2.7)-(2.8)}

We establish now an error estimate for the approximation of equations (2.7)-(2.8). For this section, we make the following assumptions on the data:

$$
\rho^{0} \in W^{1, \infty}(\Omega), \beta \in W^{1, \infty}(\Omega), N \in W^{1, \infty}(\partial \Omega), N \geq 0, \int_{\partial \Omega} N(\sigma) \mathrm{d} \sigma=1, f \in W^{1, \infty}(] 0, T[\times \partial \Omega) .
$$

It can be noticed that in order to perform the weak convergence of the approximated solutions and establish theoretical existence to the continuous problem, we did not need to approximate the characteristics $\Phi(t ; \tau, \sigma)$ of the equation. In view of the error estimate though, we need to use another approximation of $\beta(\Phi(t ; \tau, \sigma))$ than (3.3). We introduce an approximation $\Phi_{h}(t ; \tau, \sigma)$ of the characteristics given by a numerical integrator of the ODE system (1.2)-(1.3) and define

$$
\begin{gathered}
\beta_{i, j}^{1}:=\beta\left(\Phi_{h}\left(t_{k} ; \tau_{i}, \sigma_{j}\right)\right), \beta_{l, m}^{2}:=\beta\left(\Phi_{h}\left(t_{k} ; 0,\left(x_{l}, \theta_{m}\right)\right)\right. \\
f_{j}^{k}:=f\left(t_{k}, \sigma_{j}\right), N_{j}:=N\left(\sigma_{j}\right) .
\end{gathered}
$$

For $g_{1}$ and $g_{2}$ being two continuous functions on $\widetilde{Q}_{1}$ and $] 0, T[\times \Omega$ respectively, we define

$$
\begin{array}{ll}
\mathbb{P}_{1} g_{1}(t, \tau, \sigma(s))=g_{1}\left(t_{k}, \tau_{i}, \sigma_{j}\right) & \text { for } t \in\left[t_{k}, t_{k+1}[, \tau \in] \tau_{i-1}, \tau_{i}\right], s \in\left[s_{j}, s_{j+1}[\right. \\
\mathbb{P}_{1} g_{1}(t, \tau, \sigma(s))=0 & \text { for } t \in\left[t_{k}, t_{k+1}[, \tau \in] t_{k}, t\right], s \in\left[s_{j}, s_{j+1}[\right. \\
\mathbb{P}_{2} g_{2}(t, x, \theta)=g_{2}\left(t_{k}, x_{l}, \theta_{m}\right) & \text { for } t \in\left[t_{k}, t_{k+1}[, x \in] x_{l}, x_{l+1}\right], \theta \in\left[\theta_{m}, \theta_{m+1}[\right.
\end{array}
$$

Lemma 3.2 (projection error). Let $\left(g_{1}, g_{2}\right) \in W^{1, \infty}\left(\widetilde{Q}_{1}\right) \times W^{1, \infty}(] 0, T[\times \Omega)$. Then there exists $C_{\mathbb{P}_{1}}$ and $C_{\mathbb{P}_{2}}$ such that

$$
\left\|g_{1}\left(t_{k}, \cdot\right)-\mathbb{P}_{1} g_{1}\left(t_{k}, \cdot\right)\right\|_{L^{\infty}\left(j 0, t_{k}[)\right.} \leq C_{\mathbb{P}_{1}} h, \quad\left\|g_{2}\left(t_{k}, \cdot\right)-\mathbb{P}_{2} g_{2}\left(t_{k}, \cdot\right)\right\|_{L^{\infty}(\Omega)} \leq C_{\mathbb{P}_{2}} h .
$$

The proof of this lemma is straightforward from the fact that $g_{1}$ and $g_{2}$ are Lipschitz continuous. We define

$$
e_{1, h}:=\rho_{1, h}-\mathbb{P}_{1} \widetilde{\rho}_{1} \text { and } e_{2, h}:=\rho_{2, h}-\mathbb{P}_{2} \widetilde{\rho}_{2}
$$

the errors of the schemes, with $\left(\widetilde{\rho}_{1}, \widetilde{\rho}_{2}\right)$ solving the problem (2.7)-(2.8). From equation (2.8) we have

$$
\begin{cases}\widetilde{\rho}_{1}\left(t_{k+1}, \tau_{i}, \sigma_{j}\right)=\widetilde{\rho}_{1}\left(t_{k}, \tau_{i}, \sigma_{j}\right) & 0 \leq k \leq K, 0 \leq i \leq k, 1 \leq j \leq M \\ \widetilde{\rho}_{1}\left(\tau_{k+1}, \tau_{k+1}, \sigma_{j}\right)=N\left(\sigma_{j}\right) \widetilde{B}\left(\tau_{k+1}, \widetilde{\rho}_{1}, \widetilde{\rho}_{2}\right)+f\left(\tau_{k+1}, \sigma_{j}\right) & 0 \leq k \leq K, 1 \leq j \leq M\end{cases}
$$

and thus, subtracting this to $(3.1)$ and denoting $e_{1}^{k}(i, j)=e_{1, h}\left(t_{k}, \tau_{i}, \sigma_{j}\right)$ we obtain

$$
\left\{\begin{array}{l}
e_{1}^{k+1}(i, j)=e_{1}^{k}(i, j), \quad 0 \leq k \leq K, 0 \leq i \leq k, 1 \leq j \leq M \\
e_{1}^{k+1}(k+1, j)=N_{j} E^{k+1}+r_{j}^{k+1}
\end{array}\right.
$$


with

$$
\begin{aligned}
E^{k+1} & =\sum_{i=1}^{k} \sum_{j=1}^{M} \beta_{i, j}^{1} e_{1}^{k+1}(i, j) \delta t \delta \sigma+\sum_{l, m=1}^{L} \beta_{l, m}^{2} e_{2}^{k+1}(l, m)(\delta x)^{2} \\
r_{j}^{k+1} & =N_{j}\left(B^{k+1}\left(\left(\widetilde{\rho}_{1}\left(t_{k+1}, \tau_{i}, \sigma_{j}\right)\right)_{i, j},\left(\widetilde{\rho}_{2}\left(t_{k+1}, x_{l}, \theta_{m}\right)\right)_{l, m}\right)-\widetilde{B}\left(t_{k+1}, \widetilde{\rho}_{1}, \widetilde{\rho}_{2}\right)\right)
\end{aligned}
$$

Hence the truncation error of the scheme $r_{j}^{k+1}$ only comes from the quadrature error of the approximation of the integral in $\widetilde{B}\left(t_{k}, \widetilde{\rho}_{1}, \widetilde{\rho}_{2}\right)$.

Lemma 3.3 (truncation error). Assume (3.8), that $\left(\beta \circ \Phi_{1}\right) \widetilde{\rho}_{1} \in W^{1, \infty}\left(\widetilde{Q}_{1}\right),\left(\beta \circ \Phi_{2}\right) \widetilde{\rho}_{2} \in W^{1, \infty}(] 0, T[\times \Omega)$ and that the numerical integrator for the ODE system (1.2)-(1.3) is of order at least 1 . Then there exists $C_{r}$ such that

$$
\max _{k, j}\left|r_{j}^{k}\right| \leq C_{r} h
$$

Proof. We have

$$
\begin{aligned}
r_{j}^{k}= & N_{j}\left[\sum_{i=1}^{k-1} \sum_{j=1}^{M}\left(\beta_{i, j}^{1}-\beta\left(\Phi_{1}\left(t_{k} ; \tau_{i}, \sigma_{j}\right)\right)\right) \widetilde{\rho}_{1}\left(t_{k}, \tau_{i}, \sigma_{j}\right) \delta t \delta \sigma+\sum_{l, m=1}^{L}\left(\beta_{l, m}^{2}-\beta\left(\Phi_{2}\left(t_{k} ; x_{l}, \theta_{m}\right)\right)\right) \widetilde{\rho}_{2}\left(t_{k}, x_{l}, \theta_{m}\right)(\delta x)^{2}\right. \\
& +\sum_{i=1}^{k-1} \sum_{j=1}^{M} \beta\left(\Phi_{1}\left(t_{k} ; \tau_{i}, \sigma_{j}\right)\right) \widetilde{\rho}_{1}\left(t_{k}, \tau, \sigma\right) \delta t \delta \sigma+\sum_{l, m=1}^{L} \beta\left(\Phi_{2}\left(t_{k} ; x_{l}, \theta_{m}\right)\right) \widetilde{\rho}_{2}\left(t_{k}, x_{l}, \theta_{m}\right)(\delta x)^{2} \\
& -\int_{0}^{t_{k-1}} \int_{\partial \Omega} \beta\left(\Phi_{1}\left(t_{k} ; \tau, \sigma\right)\right) \widetilde{\rho}_{1}\left(t_{k}, \tau, \sigma\right) \mathrm{d} \tau \mathrm{d} \sigma-\int_{\Omega} \beta\left(\Phi_{2}\left(t_{k}, Y\right)\right) \widetilde{\rho}_{2}\left(t_{k}, Y\right) \mathrm{d} Y \\
& \left.-\int_{t_{k-1}}^{t_{k}} \int_{\partial \Omega} \beta\left(\Phi_{1}\left(t_{k} ; \tau, \sigma\right)\right) \widetilde{\rho}_{1}\left(t_{k}, \tau, \sigma\right) \mathrm{d} \tau \mathrm{d} \sigma\right]
\end{aligned}
$$

Thus

$$
\begin{aligned}
\left|r_{j}^{k}\right| \leq & \|N\|_{L^{\infty}}\left\{\|\beta\|_{W^{1, \infty}}\left(\left.\left\|\Phi_{1, h}-\mathbb{P}_{1} \Phi_{1}\right\|\right|_{L^{\infty}}\left\|\mathbb{P}_{1} \widetilde{\rho}_{1}\right\|_{L^{1}}+\left\|\Phi_{2, h}-\mathbb{P}_{2} \Phi_{2}\right\| L_{L^{\infty}}\left\|\mathbb{P}_{2} \widetilde{\rho}_{2}\right\|_{L^{1}}\right)\right. \\
& +\sum_{i=1}^{k-1} \sum_{j=1}^{M} \int_{\tau_{i-1}}^{\tau_{i}} \int_{\sigma_{j}}^{\sigma_{j+1}}\left|\mathbb{P}_{1}\left[\left(\beta \circ \Phi_{1}\right) \widetilde{\rho}_{1}\right]\left(t_{k}, \tau, \sigma\right)-\left(\beta \circ \Phi_{1}\right) \widetilde{\rho}_{1}\left(t_{k}, \tau, \sigma\right)\right| \mathrm{d} \tau \mathrm{d} \sigma \\
& \left.+\sum_{l, m=1}^{L} \int_{x_{l}}^{x_{l+1}} \int_{x_{m}}^{x_{m+1}}\left|\mathbb{P}_{2}\left[\left(\beta \circ \Phi_{2}\right) \widetilde{\rho}_{2}\right]\left(t_{k}, Y\right)-\left(\beta \circ \Phi_{2}\right) \widetilde{\rho}_{2}\left(t_{k}, x, \theta\right)\right| \mathrm{d} x \mathrm{~d} \theta+\left\|\left(\beta \circ \Phi_{1}\right) \widetilde{\rho}_{1}\right\|_{L^{\infty}} h\right\} .
\end{aligned}
$$

Using the lemma 3.2 and the $L^{1}$ a priori estimate of Proposition 2.2 gives the result.

Remark 3.2 (order of the truncation error). In order to have a better order for the truncation error we could use a more sophisticated quadrature method like for instance the trapezoid method on $\Omega$ for $\widetilde{\rho}_{2}$ and on $\left[0, t_{k-1}[\times \partial \Omega\right.$ for $\widetilde{\rho}_{1}$ (completed by a left rectangle method on $\left[t_{k-1}, t_{k}[\times \partial \Omega)\right.$. Adapting the previous proof shows that if the numerical integrator used for the characteristics has order larger than 2 , then the truncation error would have order 2 (order of the trapezoid method).

Proposition 3.2 (error estimate). Assume (3.8) and that $\left(\widetilde{\rho}_{1}, \widetilde{\rho}_{2}\right) \in W^{1, \infty}\left(\widetilde{Q}_{1}\right) \times W^{1, \infty}(] 0, T[\times \Omega)$ is a regular solution of $(2.7)-(2.8)$. Let $\rho_{1, h}$ and $\rho_{2, h}$ solve (3.1) and (3.2). Then there exists some constants $\widetilde{C}_{1}$ and $\widetilde{C}_{2}$ 
such that

$$
\left\|\rho_{1, h}\left(t_{k}, \cdot\right)-\widetilde{\rho}_{1}\left(t_{k}, \cdot\right)\right\|_{L^{1}(] 0, t_{k}[\times \partial \Omega)} \leq \widetilde{C}_{1} h, \quad\left\|\rho_{2, h}\left(t_{k}, \cdot\right)-\widetilde{\rho}_{2}\left(t_{k}, \cdot\right)\right\|_{L^{1}(\Omega)} \leq \widetilde{C}_{2} h .
$$

Proof. In view of Lemma 3.2 , it is sufficient to prove the proposition with $\mathbb{P}_{s} \widetilde{\rho}_{s}\left(t_{k}, \cdot\right)$ instead of $\widetilde{\rho}_{s}\left(t_{k}, \cdot\right)$ (with $s=1,2)$. For the second estimate, we notice that

$$
\left\|\rho_{2, h}\left(t_{k}, \cdot\right)-\mathbb{P}_{2} \widetilde{\rho}_{2}\left(t_{k}, \cdot\right)\right\|_{L^{1}(\Omega)}=\left\|e_{2, h}\left(t_{k}, \cdot\right)\right\|_{L^{1}(\Omega)}=\sum_{l, m}\left|e_{2}^{k}(l, m)\right|(\delta x)^{2}=\sum_{l, m}\left|\rho_{2}^{0}(l, m)-\rho^{0}\left(x_{l}, \theta_{m}\right)\right|(\delta x)^{2}
$$

and the result follows from the definition of $\rho_{2}^{0}(l, m)$. For the first one, we have

$$
\left\|\rho_{1, h}\left(t_{k}, \cdot\right)-\mathbb{P}_{1} \widetilde{\rho}_{1}\left(t_{k}, \cdot\right)\right\|_{L^{1}(] 0, t_{k}[\times \partial \Omega)}=\left\|e_{1, h}\left(t_{k}, \cdot\right)\right\|_{L^{1}(] 0, t_{k}[\times \partial \Omega)}=\sum_{i=1}^{k} \sum_{j=1}^{M}\left|e_{1}^{k}(i, j)\right| \delta t \delta \sigma .
$$

We can compute, using (3.12)

$$
\begin{aligned}
\left\|e_{1, h}\left(t_{k+1}, \cdot\right)\right\|_{L^{1}} & \leq \sum_{i=1}^{k} \sum_{j=1}^{M}\left|e_{1}^{k+1}(i, j)\right| \delta t \delta \sigma+\left|E^{k+1}\right| \delta t \sum_{j=1}^{M} N_{j} \delta \sigma+\delta t \sum_{j=1}^{M}\left|r_{j}^{k+1}\right| \delta \sigma \\
& \leq\left\|e_{1, h}\left(t_{k}, \cdot\right)\right\|_{L^{1}}+\delta t\|\beta\|\left\|_{\infty}\right\| N \|_{h}\left\{\left\|e_{1, h}\left(t_{k}, \cdot\right)\right\|_{L^{1}}+\left\|e_{2, h}\left(t_{k+1}, \cdot\right)\right\|_{L^{1}}\right\}+C_{r} h \delta t \\
& \leq\left(1+\delta t\|\beta\|_{\infty}\|N\| \|_{h}\right)\left\|e_{1, h}\left(t_{k}, \cdot\right)\right\|_{L^{1}}+\widetilde{C}_{2}\|\beta\|_{\infty}\|N\|_{h} h \delta t+C_{r} h \delta t
\end{aligned}
$$

and conclude using a discrete Gronwall Lemma.

Remark 3.3 (order of the error).

- By looking more carefully at the propagation of errors in the proof, we see that if we set $\rho_{2}^{0}(l, m)=\rho^{0}(l, m)$ (which is valid under (3.8)), the error on $\widetilde{\rho}_{2}$ only comes from the projection error.

- If in addition, we follow Remark 3.2 for the approximation of the data, then the error between $\rho_{1, h}$ and $\mathbb{P}_{1} \widetilde{\rho}_{1}$ would be of order 2 if we had used a trapezoid method for the integral term in $\widetilde{B}\left(t_{k}, \widetilde{\rho}_{1}, \widetilde{\rho}_{2}\right)$.

\subsection{Application to approximation of problem (1.1)}

We explain now how we approximate the solution of (1.1) from the approximation of the solutions of problems (2.7)-(2.8) given by the schemes (3.1)-(3.2). We translate formula (2.11) at the discrete level thanks to $\widetilde{\rho}_{1, h}, \widetilde{\rho}_{2, h}$ given by $(3.4)$ and the solutions $\widetilde{\rho}_{1}^{k}(i, j), \widetilde{\rho}_{2}^{k}(i, j)$ of the schemes (3.1) and (3.2) to define

$$
\rho_{h}(t, X):=\underbrace{\widetilde{\rho}_{1, h}\left(t, \tau^{t}(X), \sigma^{t}(X)\right) J_{1, h}^{-1}\left(t, \tau^{t}(X), \sigma^{t}(X)\right) \mathbf{1}_{X \in \Omega_{1}^{t}}}_{:=\rho_{1, h}}+\underbrace{\widetilde{\rho}_{2, h}(t, Y(X)) J_{2, h}^{-1}(t, Y(X)) \mathbf{1}_{X \in \Omega_{2}^{t}}}_{:=\rho_{2, h}} .
$$

The jacobians of the changes of variables $J_{1}(t ; \tau, \sigma)=|G(\tau, \sigma) \cdot \vec{\nu}(\sigma)| \mathrm{e}^{\int_{\tau}^{t} \operatorname{div} G(u, \Phi(u ; \tau, \sigma)) \mathrm{d} u}$ and $J_{2}(t ; Y)=$ $\mathrm{e}^{\int_{0}^{t} \operatorname{div} G(u, \Phi(u ; 0, Y)) \mathrm{d} u}$ are approximated respectively by $J_{1, h}$ and $J_{2, h}$, piecewise constant functions constructed similarly as in (3.4) through $J_{1}^{k}(i, j):=\mathrm{e}^{\mathcal{T}_{1}(k, i, j)}$ and $J_{2}^{k}(l, m):=\mathrm{e}^{\mathcal{T}_{2}(k, l, m)}$, where $\mathcal{T}_{1}$ and $\mathcal{T}_{2}$ are one-dimensional quadrature methods such that $\mathcal{T}_{1}(k, i, j) \simeq \int_{\tau_{i}}^{t_{k}} \operatorname{div} G\left(\Phi\left(s ; \tau_{i}, \sigma_{j}\right)\right) \mathrm{d} s$ and $\mathcal{T}_{2}(k, l, m) \simeq \int_{0}^{t_{k}} \operatorname{div} G\left(\Phi\left(s ; 0,\left(x_{l}, \theta_{m}\right)\right)\right) \mathrm{d} s$. The errors of these quadrature methods are denoted by $r_{1}, r_{2}$ and are assumed to be of order $\alpha_{1}, \alpha_{2}$ :

$$
\overline{r_{1}}:=\max _{k, i, j}\left|r_{1}(k, i, j)\right| \leq C_{q}(\delta t)^{\alpha_{1}}, \overline{r_{2}}:=\max _{k, l, m}\left|r_{2}(k, l, m)\right| \leq C_{q}(\delta t)^{\alpha_{2}} .
$$

We have

$$
J_{1}^{k}(i, j)=J_{1}\left(t_{k}, \tau_{i}, \sigma_{j}\right) \mathrm{e}^{-r_{1}(k, i, j)}, J_{2}^{k}(l, m):=\mathrm{e}^{\mathcal{T}_{2}(k, l, m)}=J_{2}\left(t_{k}, x_{l}, \theta_{m}\right) \mathrm{e}^{-r_{2}(k, l, m)} .
$$


We define the following meshes:

$$
\begin{gathered}
V_{1}(k, i, j)=\left\{(t, \Phi(t ; \tau, \sigma(s))) ; t \in\left[t_{k}, t_{k+1}[, \tau \in] \tau_{i-1}, \tau_{i}\right], s \in\left[s_{j}, s_{j+1}[\}\right.\right. \\
V_{2}(k, l, m)=\left\{\left(t, \Phi\left(t ; 0,\left(x_{l}, \theta_{m}\right)\right)\right) ; t \in\left[t_{k}, t_{k+1}\left[, x \in\left[x_{l}, x_{l+1}\left[, \theta \in\left[\theta_{m}, \theta_{m+1}[\}\right.\right.\right.\right.\right.\right.
\end{gathered}
$$

and, for a function $g \in \mathcal{C}([0, T] \times \bar{\Omega})$

$$
\mathbb{P} g(t, X)=g\left(t_{k}, \Phi\left(t_{k} ; \tau_{i}, \sigma_{j}\right)\right) \mathbf{1}_{(t, X) \in V_{1}(k, i, j)}+g\left(t_{k}, \Phi\left(t_{k} ; 0,\left(x_{l}, \theta_{m}\right)\right)\right) \mathbf{1}_{(t, X) \in V_{2}(k, l, m)}
$$

Remark 3.4. In the same way as Lemma 3.2, there exists a constant $C_{\mathbb{P}}$ such that for all function $g \in$ $W^{1, \infty}(] 0, T[\times \Omega)$

$$
\|g-\mathbb{P} g\|_{L^{1}(] 0, T[\times \Omega)} \leq C_{\mathbb{P}} h .
$$

Theorem 3.2. Suppose that $\rho \in W^{1, \infty}(] 0, T[\times \Omega)$ solves the problem (1.1) and let $\rho_{h}$ be defined by (3.14). Then there exists a constant $C$ such that

$$
\sup _{t \in[0, T]}\left\|\rho_{h}(t, \cdot)-\rho(t, \cdot)\right\|_{L^{1}(\Omega)} \leq C h
$$

Proof. In view of Remark 3.4, it is again sufficient to prove the proposition with $\mathbb{P} \rho$ instead of $\rho$. Let $t \in$ $\left[t_{k}, t_{k+1}\left[\right.\right.$, then $\left\|\rho_{h}(t, \cdot)-\mathbb{P} \rho(t, \cdot)\right\|_{L^{1}(\Omega)}=\left\|\rho_{1, h}\left(t_{k}, \cdot\right)-\mathbb{P} \rho_{1}\left(t_{k}, \cdot\right)\right\|_{L^{1}\left(\Omega_{1}^{t_{k}}\right)}+\left\|\rho_{2, h}\left(t_{k}, \cdot\right)-\mathbb{P} \rho_{2}\left(t_{k}, \cdot\right)\right\|_{L^{1}\left(\Omega_{2}^{t_{k}}\right)}$ with $\rho_{s}(t, X):=\rho(t, X) \mathbf{1}_{X \in \Omega_{s}^{t}}(s=1,2)$. We do the proof only for $\rho_{1}$ since it is similar for $\rho_{2}$. We also don't write the dependency in $\sigma$ in order to avoid heavy notations. To obtain the complete proof it suffices to add integrals with respect to $\sigma$ in the following and $\sigma$ in all the functions. Doing the change of variables $\Phi_{1}$ we have, noticing that $\mathbb{P} \rho_{1}\left(t_{k}, \Phi\left(t_{k} ; \tau\right)\right)=\mathbb{P}_{1} \widetilde{\rho}_{1}\left(t_{k}, \tau\right) \mathbb{P}_{1} J_{1}^{-1}\left(t_{k}, \tau\right)$

$$
\begin{aligned}
\left\|\rho_{1, h}\left(t_{k}, \cdot\right)-\mathbb{P} \rho_{1}\left(t_{k}, \cdot\right)\right\|_{L^{1}\left(\Omega_{1}^{t_{k}}\right)}= & \int_{0}^{t_{k}}\left|\widetilde{\rho}_{1, h}\left(t_{k}, \tau\right) J_{1, h}^{-1}\left(t_{k}, \tau\right)-\mathbb{P}_{1} \widetilde{\rho}_{1}\left(t_{k}, \tau\right) \mathbb{P}_{1} J_{1}^{-1}\left(t_{k}, \tau\right)\right| J_{1}\left(t_{k}, \tau\right) \mathrm{d} \tau \\
\leq & \int_{0}^{t_{k}}\left|\widetilde{\rho}_{1, h}\left(t_{k}, \tau\right)\right|\left|J_{1, h}^{-1}\left(t_{k}, \tau\right) J_{1}\left(t_{k}, \tau\right)-1\right| \mathrm{d} \tau \\
& +\int_{0}^{t_{k}}\left|\widetilde{\rho}_{1, h}\left(t_{k}, \tau\right)-\mathbb{P}_{1} \widetilde{\rho}_{1}\left(t_{k}, \tau\right)\right| \mathrm{d} \tau+ \\
& +\int_{0}^{t_{k}}\left|\mathbb{P}_{1} \widetilde{\rho}_{1}\left(t_{k}, \tau\right)\right|\left|1-\mathbb{P}_{1} J_{1}^{-1}\left(t_{k}, \tau\right) J_{1}\left(t_{k}, \tau\right)\right| \mathrm{d} \tau
\end{aligned}
$$

Now we have, using the definition (3.15)

$$
\left|J_{1, h}^{-1} J_{1}-1\right|=\left|\mathbb{P} J_{1}^{-1} \mathrm{e}^{-r_{1}} J_{1}-1\right| \leq\left|\mathrm{e}^{\overline{r_{1}}}\right| \frac{1}{\left|\mathbb{P} J_{1}\right|}\left|J_{1}-\mathbb{P} J_{1}\right|+\left|\mathrm{e}^{-r_{1}}-1\right| .
$$

Thus, since $\left\|\frac{1}{J_{1}}\right\|_{L^{\infty}}<\infty$ from formula (2.5) and the fact that $-G \cdot \nu \geq \delta>0$, and using $\left|\mathrm{e}^{-r_{1}}-1\right| \leq 2 \overline{r_{1}}$, there exists $C_{J}$ such that

$$
\left\|J_{1, h}^{-1}\left(t_{k}, \tau\right) J_{1}\left(t_{k}, \tau\right)-1\right\|_{L^{\infty}} \leq C_{J} h, \text { and }\left\|1-\mathbb{P}_{1} J_{1}^{-1}\left(t_{k}, \tau\right) J_{1}\left(t_{k}, \tau\right)\right\|_{L^{\infty}} \leq C_{J} h .
$$

The last inequality comes from Lemma 3.2 since $J_{1} \in W^{1, \infty}$ from the formula (2.5). Using then the continuous and discrete a priori $L^{1}$ estimates and Proposition 3.2 gives the result. 
Remark 3.5. In the case of less regularity on the solution, we still have $\rho_{h} \underset{h \rightarrow 0}{\rightarrow} \rho, *-L^{\infty}(] 0, T[\times \Omega)$. Indeed, we write $\rho_{h}=\widetilde{\rho}_{1, h} J_{1, h}^{-1}+\widetilde{\rho}_{2, h} J_{2, h}^{-1}=\widetilde{\rho}_{1, h} J_{1}^{-1}+\widetilde{\rho}_{2, h} J_{2}^{-1}+\widetilde{\rho}_{1, h}\left(J_{1, h}-J_{1}\right)+\widetilde{\rho}_{2, h}\left(J_{2, h}-J_{2}\right)$. Then we use that for $s=1,2 J_{s, h}^{-1} \underset{h \rightarrow 0}{\stackrel{L^{1}}{\longrightarrow}} J_{s}^{-1}$ as well as $\left\|J_{s, h}^{-1}\right\|_{L^{\infty}} \leq C \mathrm{e}^{\bar{r}_{s}}$ with $C$ a constant. Using Theorem 3.1 for the convergence of $\widetilde{\rho}_{1, h}$ and $\widetilde{\rho}_{2, h}$ gives the result.

Remark 3.6. In practical situations we are often only interested in the number of metastases and not in the density $\rho$ itself. Thanks to the formula $\int_{\Omega} \rho(t, X) \mathrm{d} X=\int_{0}^{t} \int_{\partial \Omega} \widetilde{\rho}_{1}(t, \tau, \sigma) \mathrm{d} \sigma \mathrm{d} \tau+\int_{\Omega} \rho^{0}(X) \mathrm{d} X$, we don't have to compute the jacobians $J_{1}, J_{2}$ to get the number of metastases. Yet, we still have to compute the characteristics since they are requested in the computation of the boundary condition (see formula (3.9)).

\subsection{Numerical illustration of the accuracy of the scheme}

\subsubsection{Analytical solution}

The computational cost of a reference solution on a very fine grid is very high. Therefore, since we don't have an analytical expression of the characteristics associated to the vector field $G$ of our model defined by (1.2)-(1.3), we illustrate the accuracy of our scheme for $G$ given by

$$
G_{a}(x, \theta)=\left(\begin{array}{c}
a x \ln \left(\frac{\theta}{x}\right) \\
a \theta\left(1-\frac{\theta}{K}\right)
\end{array}\right)
$$

with $a$ and $K$ two parameters whose values are fixed to $a=0.192, K=5000$. This field has a similar phase plan to the one of our model and we can derive an analytical expression for the associated characteristics, given by

$$
\begin{aligned}
& \theta(t)=\frac{\sigma_{\theta} K}{\left(K-\sigma_{\theta}\right) \mathrm{e}^{-a t}+\sigma_{\theta}} \\
& x(t)=\mathrm{e}^{y(t)}, y(t)=\mathrm{e}^{a t}\left(\sigma_{y}+\ln (\theta(t))\left(\mathrm{e}^{a t}+\frac{K}{\sigma_{\theta}}-1\right)+a t\left(1-\frac{K}{\sigma_{\theta}}\right)-\frac{K}{\sigma_{\theta}} \ln \left(\sigma_{\theta}\right)\right)
\end{aligned}
$$

with $\sigma=\left(\sigma_{x}, \sigma_{\theta}\right)$ the starting point of the trajectory at time 0 and $\sigma_{y}=\ln \left(\sigma_{x}\right)$.

The nonlocal term of the boundary condition can be handled by taking $\beta$ constant equal to 1 and so is taken the source term $f(t)$. We take a null initial condition and thus consider the following problem

$$
\left\{\begin{array}{l}
\partial_{t} \rho+\operatorname{div}\left(\rho G_{a}\right)=0 \\
-G_{a} \cdot \nu(\sigma) \rho(t, \sigma)=N(\sigma)\left\{\int_{\Omega} \rho(t, X) \mathrm{d} X+1\right\} \\
\rho(0)=0
\end{array}\right.
$$

We can compute explicitly the integral of the solution, given by $\int_{\Omega} \rho(t, X)=\mathrm{e}^{t}-1$ so that we are able to derive an analytical expression for $\widetilde{\rho}_{a}(t, \tau, \sigma)=\rho_{a}(t, \Phi(t ; \tau, \sigma)) J_{a}(t, \tau, \sigma)$, with $\rho_{a}$ solving (3.18), $\Phi_{a}$ the change of variable defined explicitly from (3.17) corresponding to $\Phi_{1}$ from Section 2.1 and its associated jacobian

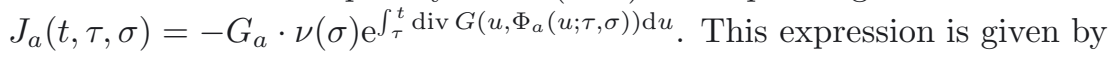

$$
\widetilde{\rho}_{a}(t, \tau, \sigma)=N(\sigma) \mathrm{e}^{\tau} \mathbf{1}_{\tau \leq t}
$$

so that to get the reference solution $\rho_{a}(t, \Phi(t ; \tau, \sigma))=\widetilde{\rho}_{a} J_{a}^{-1}(t, \tau, \sigma)$ we only need to finely approximate the jacobian, which is numerically tractable since it involves only the approximation of a $1 \mathrm{D}$ integral and that we have an exact expression for $\Phi_{a}$. We use a trapezoid method and a timestep $\mathrm{d} t=5 \times 10^{-4}$ to achieve this. 


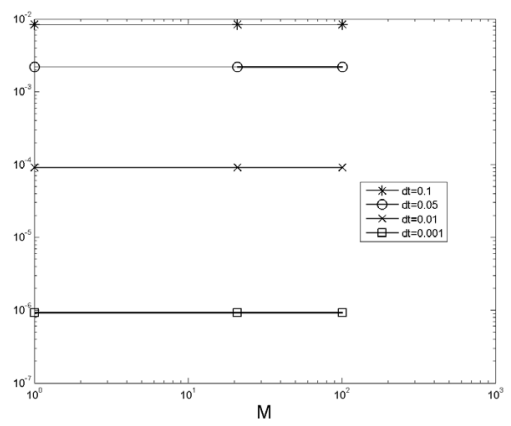

(A)

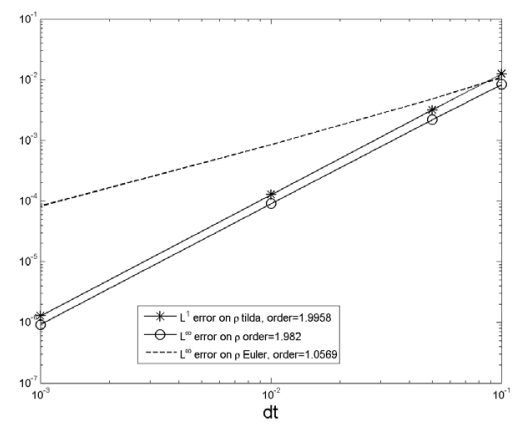

(B)

FIgURE 4. Numerical illustration of the convergence of the scheme. (A) $L^{\infty}$ error on $\rho$ plotted versus $M$, for different values of $\delta t$. (B) Various errors plotted versus $\delta t$, with $M=1$.

\subsubsection{Accuracy of the scheme}

From the proof of the error estimates (Prop. 3.2 and Thm. 3.2) we see that the error on $\widetilde{\rho}$ can be split into: (a) an error associated to the discretization of the nonlocal boundary condition, (b) an error coming from the numerical integrator used for the characteristics and (c) a projection error, whereas the error on $\rho$ has an additional term coming from the approximation of the jacobian (see Sect. 3.5). We will not consider the projection error and are aware that taking $\beta$ constant will cancel the error of the numerical integrator impacting on the boundary condition. However, this error is still present in the approximation of the jacobian and thus in the error on $\rho$ and we are more focused on the error deriving from the approximation of the nonlocal boundary condition. In Figure 4 are presented various illustrations of the convergence of the scheme for the following errors, with $T=1$ :

$$
L^{1} \text { error on } \widetilde{\rho}=\left\|\widetilde{\rho}_{h}(T, \cdot)-\widetilde{\rho}_{a}(T, \cdot)\right\|_{L^{1}(] 0, T[\times \partial \Omega)}, L^{\infty} \text { error on } \rho=\left\|\rho_{h}(T, \cdot)-\rho_{a}(T, \cdot)\right\|_{L^{\infty}(\Omega)} .
$$

Following Remark 3.3, we use a trapezoid method (completed by a left rectangle method on $\left[t_{k-1}, t_{k}[\times \partial \Omega\right.$ ) for the approximation of the integral in the boundary condition and also for the integral intervening in the jacobian. We consider a Runge-Kutta method of order 4 for the discretization of the characteristics. In Figure $4 \mathrm{~A}$, we observe that varying $M$ (number of discretization points of the boundary) with fixed $\delta t$ does not affect the error. Indeed, this comes from the facts that $N(\sigma)$ is a constant function and that we don't consider the projection error. Considering a nonconstant function for $N(\sigma)$ (like a gaussian one for instance) does produce an impact of $M$ on the error in $L^{1}$ norm (data not shown), but since we approximate the solution along each characteristic, the $L^{\infty}$ error resulting from the discretization of the boundary can only be seen between the exact solution and its projection on the mesh, that we don't consider here. In view of these consideration, we investigate the order of convergence keeping only one characteristic and varying $\delta t$.

As shown in Figure 4B we obtain convergence of order two for both the $L^{1}$ error on $\widetilde{\rho}$ and the $L^{\infty}$ error on $\rho$, in agreement with Remark 3.3. The use of an Euler scheme for the characteristics is also investigated and leads to an order 1 on the $L^{\infty}$ error on $\rho$, in concordance with the fact that the numerical integrator is used in the approximation of $\rho$ via approximation of the jacobian. Concerning $L^{1}$ error on $\widetilde{\rho}$ with Euler we have order 2 (data not shown).

\section{Numerical Simulations}

Since our equation is two-dimensional, the computational cost can be relatively high because of the integral term in the boundary, especially for large-time simulations. We use here the considerations explained in the modeling part and proved in [8] consisting in replacing $N$ by a Dirac measure in $\left(1, \theta_{0}\right)$ to reduce the computational cost and simulate only along the characteristic passing through $\left(1, \theta_{0}\right)$, that is to say the scheme (3.1) 
TABLE 1. Values of the parameters without treatment.

\begin{tabular}{|c|c|c|c|c|c|c|}
\hline $\begin{array}{c}a \\
\left(\operatorname{day}^{-1}\right)\end{array}$ & $\begin{array}{c}c \\
\left(\text { day }^{-1}\right)\end{array}$ & $\begin{array}{c}d \\
\left(\text { day }^{-1} \text { vol }^{-2 / 3}\right)\end{array}$ & $\begin{array}{l}x_{0}(\text { initial } x) \\
(\operatorname{vol})\end{array}$ & $\begin{array}{c}\theta_{0}(\text { initial } \theta) \\
(\text { vol })\end{array}$ & $\begin{array}{c}m \\
(\text { Nb of meta })\left(\text { day }^{-1}\right)\left(\text { vol }^{-\alpha}\right)\end{array}$ & $\alpha$ \\
\hline 0.192 & 5.85 & $8.73 \times 10^{-3}$ & $10^{-6}$ & 625 & $10^{-3}$ & $2 / 3$ \\
\hline
\end{tabular}

TABLE 2. Variation of the number of metastases with respect to $m$.

\begin{tabular}{|c|c|c|c|}
\hline & $M I(1.5)$ & $M I(7.5)$ & $M I(15)$ \\
\hline$m=10^{-4}$ & $5.80 \times 10^{-3}$ & $6.60 \times 10^{-2}$ & $2.79 \times 10^{-1}$ \\
\hline$m=10^{-3}$ & $5.80 \times 10^{-2}$ & $6.60 \times 10^{-1}$ & 2.81 \\
\hline$m=10^{-2}$ & $5.80 \times 10^{-1}$ & 6.62 & 30.1 \\
\hline
\end{tabular}

with only one discretization point $\sigma_{0}=\left(1, \theta_{0}\right)$ on $\partial \Omega$. We use a Runge-Kutta method of order 4 for the approximation of the characteristics and a trapezoid method for the approximation of the boundary condition.

The values of the parameters for the tumoural growth are taken from [17], where they were fitted to mice data. Following [2,19], we take $\alpha=2 / 3$ and fix the value of $m$ arbitrarily (see (1.6) for the definition of these parameters). The values of the parameters (without the treatment) are gathered in Table 1 . The size (= volume) is expressed in $\mathrm{mm}^{3}$ though until now it was expressed in number of cells. The conversion rule we use is $1 \mathrm{~mm}^{3} \simeq 10^{6}$ cells.

\subsection{Simulations without treatment}

A very important issue for clinicians is to determine the number of metastases which are not visible with medical imaging techniques (micro-metastases). Having a model for the density of metastases structured in size allows us to compute the number of visible and non-visible metastases. We took as threshold for a metastasis to be visible a size of $10^{8}$ cells, that is $100 \mathrm{~mm}^{3}$. In Figure 5, we plotted the result of a simulation showing both the total number of metastases as well as only the visible ones. We observe that at day 20 the model predicts approximately one metastasis though it is not visible. At the end of the simulation, the total number of metastases is much bigger than the number of visible ones. Notice the exponential behaviour of the total number of metastases, in accordance with the asymptotic behaviour studied in [7] in the case without treatment, since we can deduce from what is proven in this paper that there exists two constants $C_{1}, C_{2}>0$ such that

$$
C_{1} \mathrm{e}^{\lambda_{0} t} \leq \int_{\Omega} \rho(t, X) \mathrm{d} X \leq C_{2} \mathrm{e}^{\lambda_{0} t}
$$

where the Malthus parameter $\lambda_{0}$ satisfies a spectral equation.

Thus, an interesting application of the model would be to help designing a predictive tool for the total number of metastases present in the organism of the patient. In this perspective, we define a metastatic index as the integral of $\rho$ on $\Omega$ :

$$
M I(t):=\int_{\Omega} \rho(t, X) \mathrm{d} X .
$$

Of course, this index depends on the values of the parameters, for example on the parameter $m$, as shown in Table 2. The larger $m$, the larger the metastatic index. In this table, we remark that at least for times less than 15 days, it seems that the metastatic index is linear in $m$. Indeed, this can be explained by the fact that at the beginning, most of the metastases come from the primary tumour and not by the metastases themselves (see Fig. 6A). This means that the renewal term in the boundary condition of (1.1) could be neglected for small 


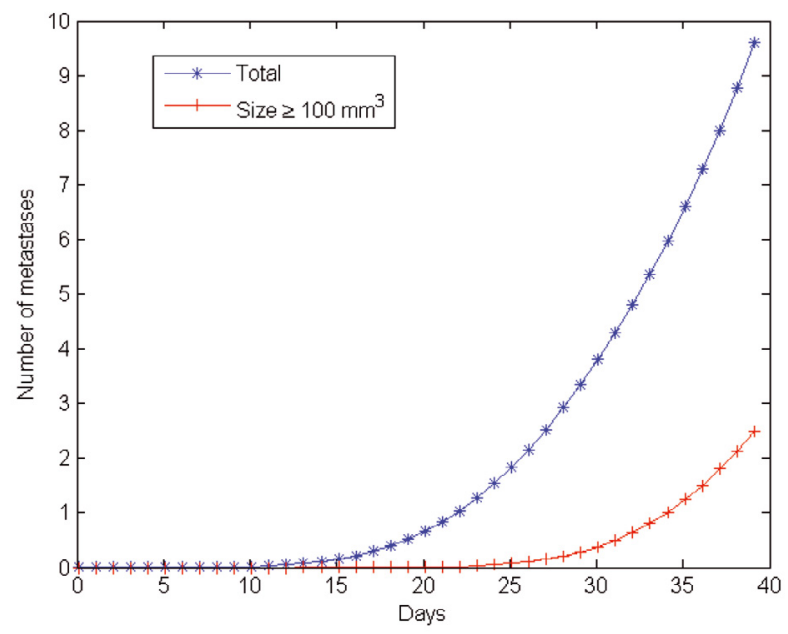

Figure 5. Evolution of the total number of metastases and of the number of visible metastases, that is whose size is bigger than $100 \mathrm{~mm}^{3}\left(\simeq 10^{8}\right.$ cells).

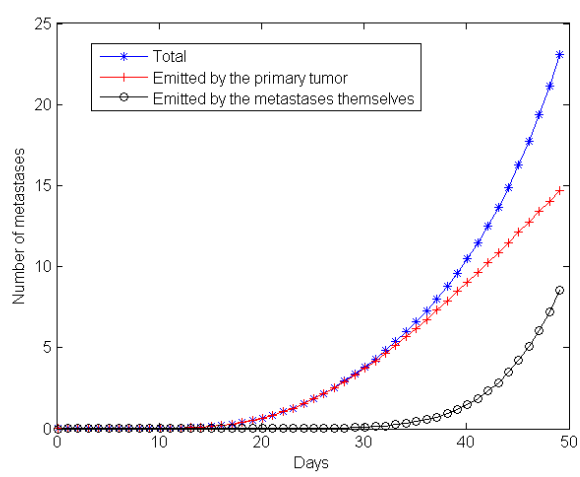

(A)

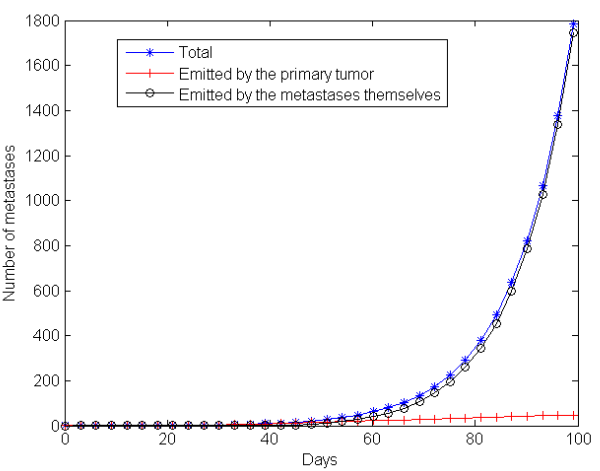

(B)

Figure 6 . Number of metastases emitted by the primary tumour and by the metastases themselves. (A) $T=50$. (B) $T=100$.

times and that the solution of (1.1) is close to the one of

$$
\left\{\begin{array}{l}
\partial_{t} \rho+\operatorname{div}(\rho G)=0 \\
-G \cdot \nu \rho(t, \sigma)=N(\sigma) \beta\left(X_{p}(t)\right) \\
\rho^{0}(X)=0
\end{array}\right.
$$

But then, integrating the equation on $\Omega$ gives $M I(t)=\int_{0}^{t} \beta\left(X_{p}(s)\right) \mathrm{d} s=m \int_{0}^{t} x_{p}(s)^{\alpha} \mathrm{d} s$, where $X_{p}(s)=$ $\left(x_{p}(s), \theta_{p}(s)\right)$ represents the primary tumour and solves the system $(1.2)-(1.3)$ with initial condition $\left(x_{0}, \theta_{0}\right)$. Figure $6 \mathrm{~B}$ shows that for larger times metastases emitted by the metastases themselves are more important than the ones emitted by the primary tumour. The metastatic index for large time is then not anymore linear in $m$, neither exponential (result not shown). 


\subsection{Simulations with treatment}

\subsubsection{Anti-angiogenic drug alone}

. We present various simulations of treatments, first involving an anti-angiogenic drug (AA) alone, in order to investigate the difference in effectiveness of various drugs regarding to their pharmacokinetic/pharmacodynamic parameters. The first result shown in Figure 7 takes the three drugs which were used in [17] where only the effect on tumour growth was investigated, and simulates the effect on the metastases. The three drugs are TNP-470, endostatin and angiostatin and each drug is characterized by two parameters in the model: its efficacy $e$ and its clearance rate $c l r_{A}$, defined in the expressions (1.3) and (1.4). These parameters were retrieved in [17] by fitting the model to mice data. The administration protocols are the same for endostatin and angiostatin (20 mg every day) but for TNP-470 the drug is administered with a dose of $30 \mathrm{mg}$ every two days. We observe that TNP-470 seems to have the poorest efficacy due to its large clearance. As noticed in [17], the ratio $e / c l r_{A}$ should govern the efficacy of the drug and its value is 0.13 for TNP-470 and 0.39 for both endostatin and angiostatin. The model we developed is now able to simulate efficacy of the drugs on the metastatic evolution (Fig. 7C).

Interestingly, the drug which seems to be more efficient regarding to the tumour size at the end of the simulation (day 15), namely angiostatin, is not the one which gives the best result on the metastases. Indeed, the lower efficacy of endostatin regarding to ultimate size is due to a relatively high clearance provoking a quite fast rebound of the angiogenic capacity once the treatment stops. But since the tumour size was lower for longer time, the metastatic evolution was better contained. This shows that the model could be a helpful tool for the clinician since the response to a treatment can differ from the primary tumour to metastases, but the clinician has no data about micro-metastases which are not visible with imagery techniques.

. One of our main postulate in the treatment of cancer is that for a given drug, the effect can vary regarding to the temporal administration protocol of the drug, due to the combination of the pharmacokinetic of the drug and the intrinsic dynamic of tumoural and metastatic growth. To investigate the effect of varying the administration schedule of the drug, we simulated various administration protocols for the same drug (endostatin). The results are presented in Figure 8. We gave the same dose and the same number of administrations of the drug but either uniformly distributed during 10 days (endostatin 2), concentrated in 5 days (endostatin 1) or in 2 days and a half (endostatin 3).

We observe that the tumour is better stabilized with a uniform administration of the drug (endostatin 2) but the number of metastases is better reduced with the intermediate protocol (endostatin 1). It is interesting to notice that again if we look at the effects at the end of the simulation, the results are different for the tumour size and for the metastases. The two protocols endostatin 1 and endostatin 2 give the same size at the end, but not the same number of metastases. Moreover, the best protocol regarding to minimization of the final number of metastases (endostatin 1) is neither the one which provoked the largest regression of the tumour during the treatment (endostatin 3) nor the one with the most stable tumour dynamic (endostatin 2). In Figure 9, we investigate the influence of the AA dose (parameter $D_{A}$ ) on tumoural, vascular and metastatic evolution. We observe that the model is consistent since it exhibits a monotonous response to variation of the dose.

\subsubsection{Combination of anti-angiogenic and cytotoxic drug}

An important question in clinical oncology is to determinate how to combine a cytotoxic drug (CT) that kills the proliferative cells and an anti-angiogenic (AA) drug which acts on the angiogenic process, either by blocking the angiogenic factors like VEGF (monoclonal antibodies, e.g. Bevacizumab) or by inhibiting the receptors to this molecule. The AA drugs are classified as part of the cytostatic drugs as they aim at stabilizing the disease. For instance, in the treatment of breast cancer, patients which express the receptor HER receive a combination of Docetaxel (CT) and Herceptine (a tyrosine kinase inhibitor, AA). Two questions are still open: which drug should come before the other and then what is the best temporal repartition for each drug? Here, we perform a brief in silico study of the first question. Since we don't have real parameters for the cytotoxic drug we fix arbitrarily the value of each parameter $h, c l r_{C}$ and $D_{C}$ to 1 , and perform simulations of the model to investigate combination of the CT and the AA. In Figure 10, we present the results of two simulations: one giving the AA before the CT (Fig. 10A) and the other one doing the opposite (Fig. 10B). 


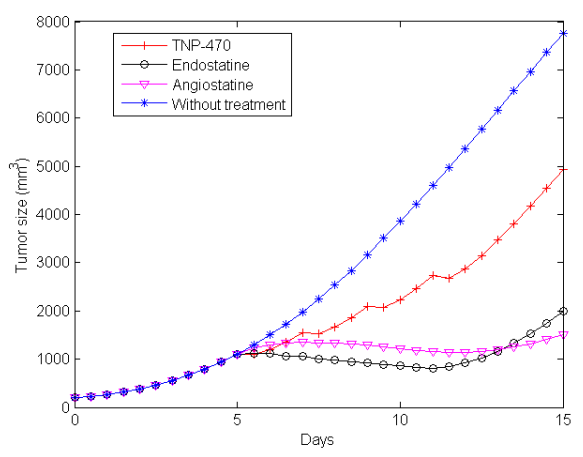

(A)

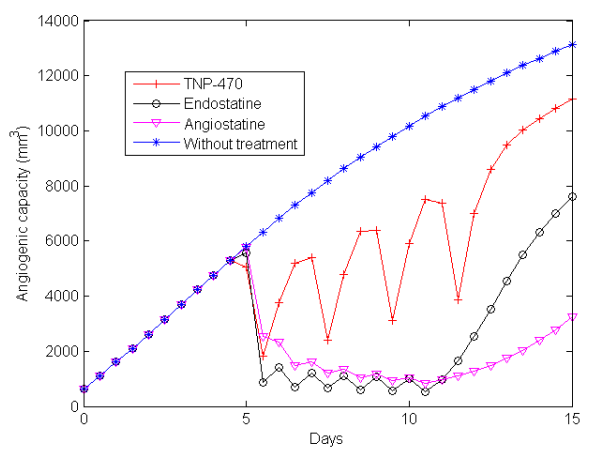

(B)

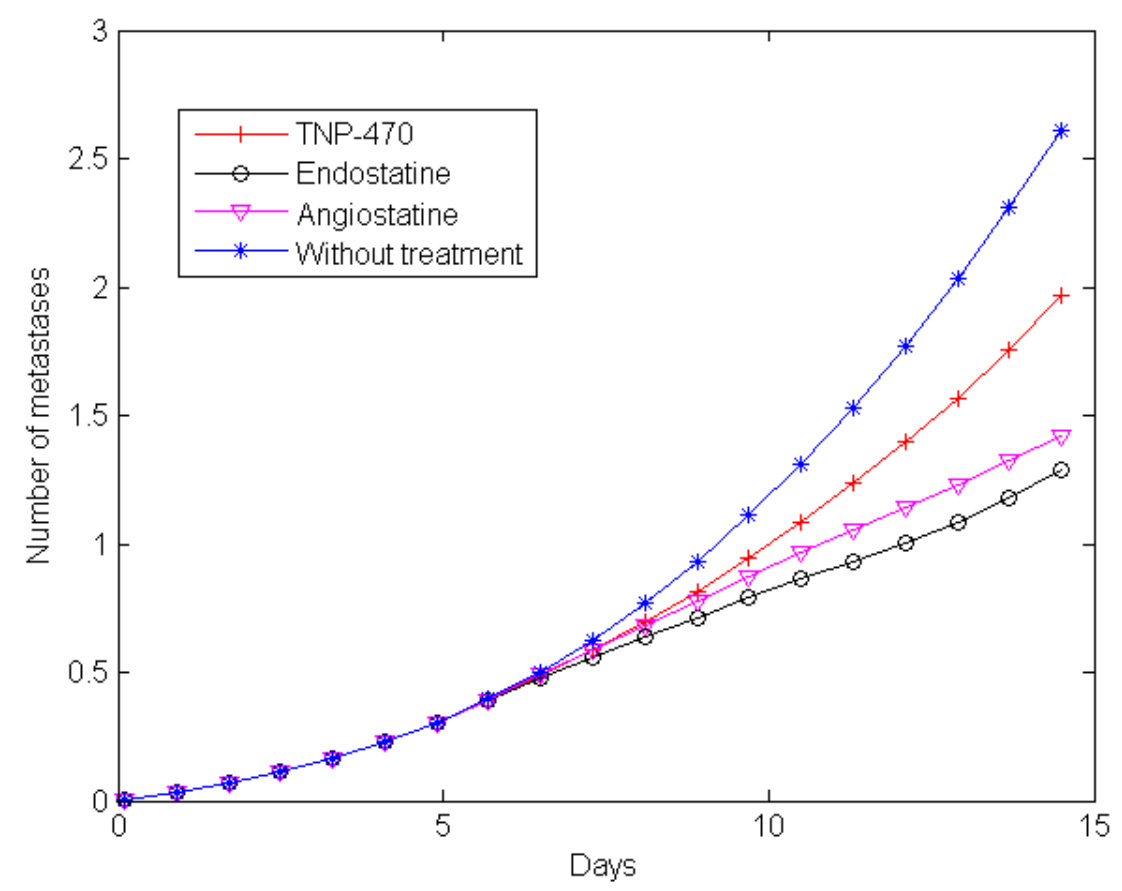

$(\mathrm{C})$

FiguRE 7. Effect of the three drugs from [17]. The treatment is administered from days 5 to 10. Endostatin $\left(e=0.66, c l r_{A}=1.7\right) 20 \mathrm{mg}$ every day, TNP-470 $\left(e=1.3, c l r_{A}=10.1\right) 30 \mathrm{mg}$ every two days and Angiostatin $\left(e=0.15, c l r_{A}=0.38\right) 20 \mathrm{mg}$ every day. (A) Tumour size. (B) Angiogenic capacity. (C) Number of metastasis.

Although in both cases the effect on the metastases is very good since the growth seems stopped (Fig. 10D), it appears that the qualitative behaviors of the tumoural and metastatic responses are different regarding to the order of administration of the drugs (Figs. 10C and 10D). According to the model, it would be better to administrate first the $\mathrm{CT}$ in order to reduce the tumour burden and then use the AA to stabilize the disease. Indeed, the number of metastasis at the end of the simulation is lower when the CT is applied first than in the opposite case. Of course, this conclusion depends on the tumoural growth and drugs parameters but this simulation shows that the model is able to exhibit different responses regarding to the order of administration between $\mathrm{CT}$ and AA drugs. 

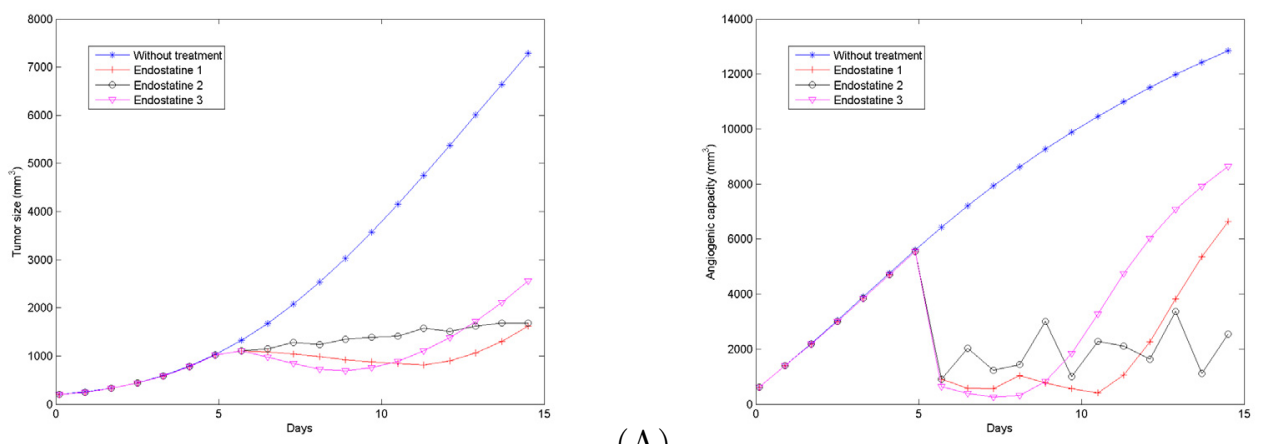

(A)

(B)

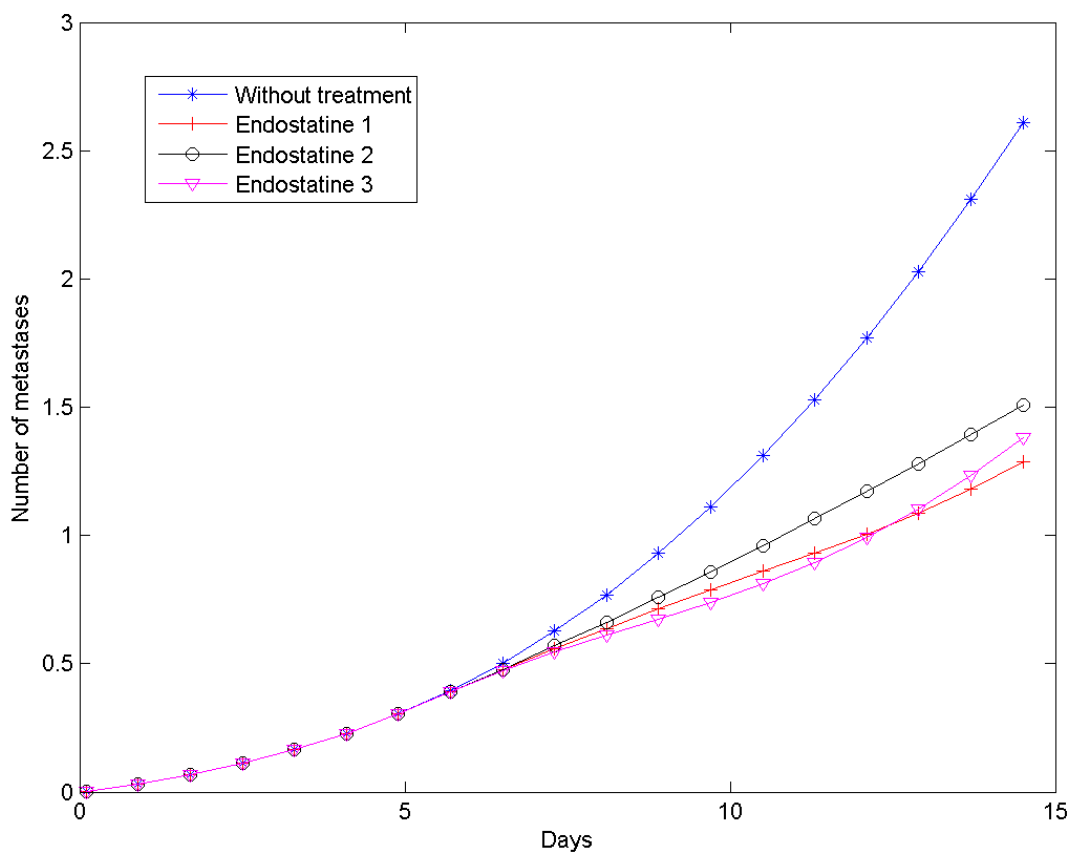

(C)

FiguRE 8. Three different temporal administration protocols for the same drug (Endostatin). Same dose $(20 \mathrm{mg}$ ) and number of administrations (6) but more or less concentrated at the beginning of the treatment. Endostatin (1) each day from day 5 to 10. Endostatin (2) every two days from day 5 to 15 . Endostatin (3) twice a day from day 5 to 7.5. (A) Tumour size. (B) Angiogenic capacity. (C) Number of metastasis.

\section{Conclusion}

In this paper, we combined the models of $[17,19]$ to obtain a model aiming at describing the effect of antiangiogenic drugs on the metastatic growth. We established the well-posedness of the model and developed an efficient numerical scheme to perform simulations, which could be adapted to similar models in higher dimensions. The model can now be used in order to rationalize the temporal administration of the antiangiogenic drugs. To achieve this, we have to implement the various pharmacokinetic models of the different AA drugs and then compare the in silico predictions to real patient data. 

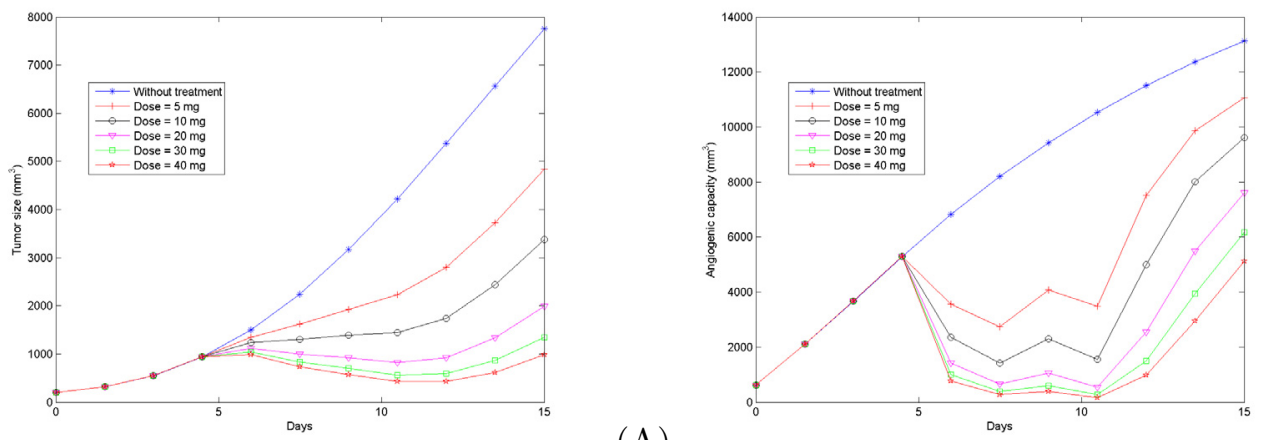

(A)

(B)

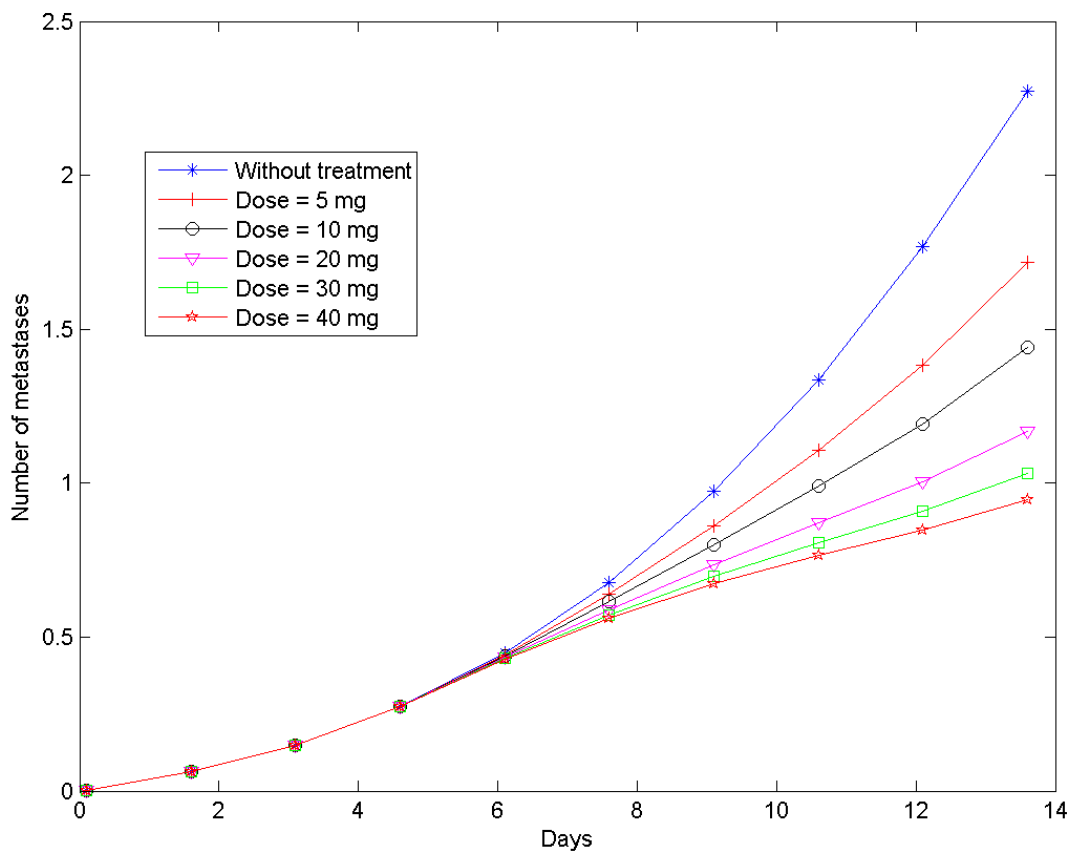

$(\mathrm{C})$

Figure 9. Effect of the variation of the dose for endostatin. (A) Tumour size. (B) Angiogenic capacity. (C) Number of metastasis.

An important open problem in this direction is the mathematical parameter identifiability of the model, that is to say the inverse problem of uniqueness of the parameters resulting in a given observation. It is also important to develop efficient numerical methods able to achieve the parameter identification from the data. Indeed, identifying the parameters $m$ and $\alpha$ in a given patient could determine the metastatic aggressiveness of its cancer, through the metastatic index. This could lead to interesting clinical applications such as a refinement of the existing classifications like TNM or SBR, which deal only with the visible metastases.

Another interesting problem would be to study the asymptotic behaviour of the solutions of the equation (1.1) in the case of periodic therapy, since it has only been studied for the autonomous case (in [7]).

As shown in [15], the metastatic response to AA treatment depends on the time schedule of the drug. The results of the simulations are encouraging in the perspective of using the model as a tool able to test various real temporal administration protocols of the drugs and to perform predictions of the mathematically optimized 

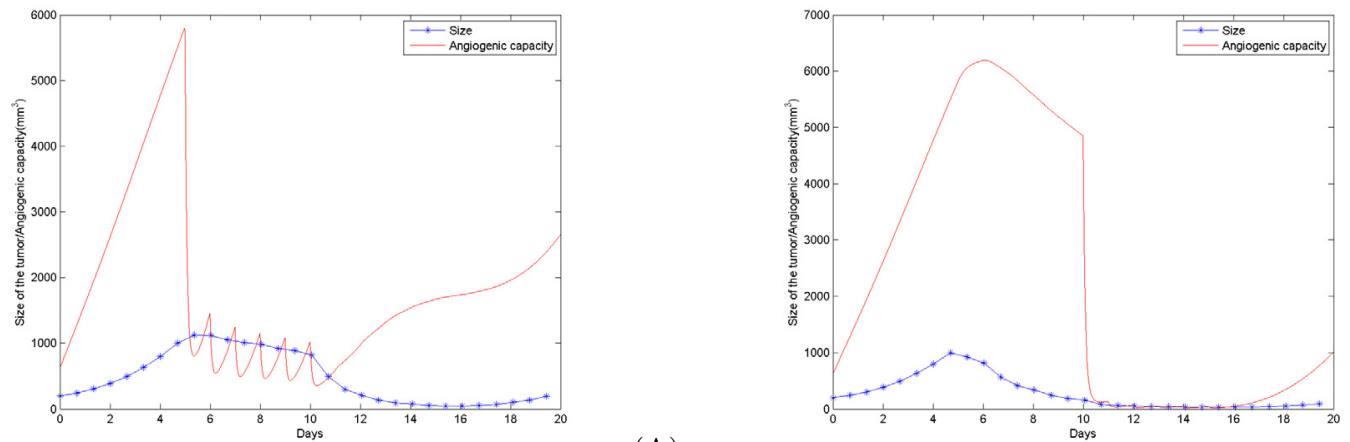

(A)

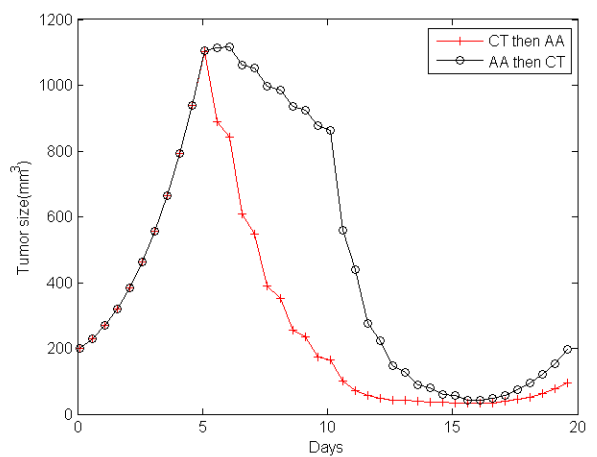

(C)

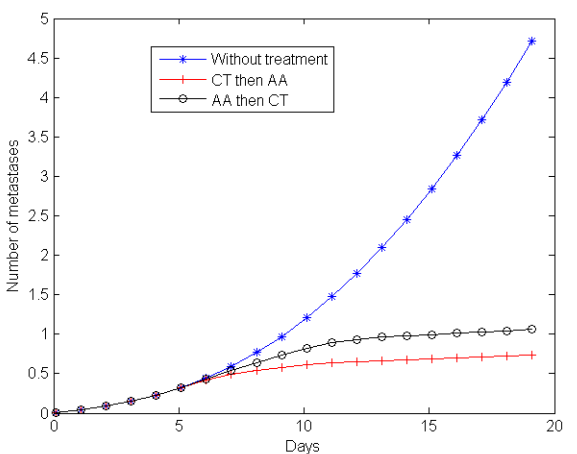

Figure 10. Combination of an anti-angiogenic drug (AA): endostatin $\left(e=0.66, \operatorname{clr}_{A}=1.7\right.$ and $D_{A}=20 \mathrm{mg}$ ) and a cytotoxic one (CT). The parameters for the CT are: $h=1, c l r_{C}=1$, and $D_{C}=1$. (A) AA from day 5 to 10 then CT from day 10 to 15, every day. Tumour growth and angiogenic capacity. (B) CT from day 5 to 10 then AA from day 10 to 15, every day. Tumour growth and angiogenic capacity. (C) Comparison between both combinations on the tumour growth. (D) Comparison between both combinations on the metastatic evolution.

schedule for a given drug. Moreover, AA are never used in a monotherapy but rather combined with a cytotoxic drug, and determining the best way to combine both drugs is still a clinical open question [24]. As shown in Figure 10, the model could help in this direction, regarding both tumour regression and metastatic evolution of the disease. We should also develop further the modeling in order to take into account the competition effects between CT and AA. Indeed, by reducing the vasculature AA drugs should induce worse supply of both drugs and on the contrary some arguments are expressed in favor of a normalization effect on the tumour vasculature by AA therapy [20], at least at the beginning of the treatment. These elements should be incorporated to the model via nonlinear terms involving the drugs concentrations in equations (1.2)-(1.3). The relative simplicity of the model (6 parameters without treatment) is a great advantage in view of concrete applications since we have to be able to fit the model to patients' data in order to retrieve their parameters and then perform predictions about the optimal schedule.

A fundamental problem that we have to integrate in our model is the one of toxicities which have to be dynamically controlled to optimize the scheduling of the drug. In the case of CT and on the tumoural growth, a model dealing with hematological toxicities is used to drive phase I clinical trials [6,27]. In our case, we also have to integrate a module to control the toxicity and address the resulting problem of optimization under constraints. 
Eventually, our model can be used to run in silico tests about the paradigm of metronomic chemotherapies which consists in delivering a cytotoxic drug at low doses and uniformly distributed in the treatment cycle rather than administrating the maximum tolerate dose (MTD) at the beginning of the cycle. Indeed, these metronomic protocols seem to have a dynamical anti-angiogenic effect $[3,18]$ that can be integrated in the model of [17] for the tumour growth and in our model for the effect on metastases.

\section{A. Proof of the Proposition 2.1}

The result for the second map is classical. For the first one, we have to deal with irregular points of the boundary $\partial \Omega$. We denote by $\chi$ the set of such points and set $\chi_{t}:=\{\Phi(t ; \tau, \xi) ; \xi \in \chi, 0 \leq \tau \leq t\}$. In order to prove the result, it is sufficient to prove that for each fixed $t$ the map

$$
\Phi_{1}^{t}: \begin{array}{ccc}
] 0, t[\times \partial \Omega \backslash \chi & \rightarrow & \Omega_{1}^{t} \backslash \chi_{t} \\
(\tau, \sigma) & \mapsto & \Phi(t ; \tau, \sigma)
\end{array}
$$

is a diffeomorphism, that globally the map $\Phi_{1}^{t}:[0, t] \times \partial \Omega \rightarrow \bar{\Omega}_{1}^{t}$ is bilipschitz and that its inverse is $X \mapsto$ $\left(\tau^{t}(X), \sigma^{t}(X)\right)$. For the first point, since we avoid the irregular points of the boundary by excluding the set $\chi$, we have the $C^{1}$ regularity. It remains to prove that $\Phi_{1}^{t}(\tau, \sigma)$ is one-to-one and onto, and that its inverse is $C^{1}$.

- The map $\Phi_{1}^{t}$ is one-to-one and onto. Let $t>0$ and $X \in \Omega_{1}^{t}$. We have $\Phi_{1}^{t}\left(\tau^{t}(X), \sigma^{t}(X)\right)=\Phi\left(t ; \tau^{t}(X)\right.$, $\left.\sigma^{t}(X)\right)=\Phi\left(t ; \tau^{t}(X), \Phi\left(\tau^{t}(X) ; t, X\right)\right)=\Phi(t ; t, X)=X$.

For the injectivity, we remark that if we have $\Phi(t ; \tau, \sigma)=\Phi\left(t ; \tau^{\prime}, \sigma^{\prime}\right)$ with for instance $\tau^{\prime}<\tau$, then $\sigma=$ $\Phi\left(\tau ; \tau^{\prime}, \sigma^{\prime}\right)$ which is prohibited by the assumption that $G \cdot \nu(\tau, \sigma)<0$. Thus $\Phi_{1}^{t}$ is one-to-one and we have, for $(\tau, \sigma) \in[0, t] \times \partial \Omega: \Phi\left(t ; \tau^{t}\left(\Phi_{1}^{t}(\tau, \sigma)\right), \sigma\left(\Phi_{1}^{t}(\tau, \sigma)\right)\right)=\Phi(t ; \tau, \sigma)$ which implies $\tau^{t}\left(\Phi_{1}^{t}(\tau, \sigma)\right)=\tau$. Thus, we have proven that the inverse of $\Phi_{1}^{t}$ is $X \mapsto\left(\tau^{t}(X), \sigma^{t}(X)\right)$.

- The map $\Phi_{1}^{t}$ is a diffeomorphism. We will prove the formula (2.5) for $J_{1}$ which will conclude the proof by using the local inversion theorem. We have $J_{1}(t ; \tau, \sigma)=\left|\partial_{\tau} \Phi_{1}^{t} \wedge \partial_{\sigma} \Phi_{1}^{t}\right|$, with $\partial_{\sigma} \Phi_{1}^{t}:=D_{Y} X \circ \sigma^{\prime}$ for $\sigma$ being a parametrization of $\partial \Omega$ and $D_{Y} X \in \mathcal{M}_{2}(\mathbb{R})$ the derivative in $Y$ of $\Phi(t ; \tau, Y)$ viewed as the flow on $\bar{\Omega}$. We compute

$$
\begin{aligned}
\partial_{t}\left(\partial_{\tau} \Phi_{1}^{t} \wedge \partial_{\sigma} \Phi_{1}^{t}\right) & =\partial_{\tau} \partial_{t} \Phi_{1}^{t} \wedge \partial_{\sigma} \Phi_{1}^{t}+\partial_{\tau} \Phi_{1}^{t} \wedge \partial_{t}\left(D_{Y} \Phi_{1}^{t} \circ \sigma^{\prime}\right)=\partial_{\tau}\left(G \circ \Phi_{1}^{t}\right) \wedge \partial_{\sigma} \Phi_{1}^{t}+\partial_{\tau} \Phi_{1}^{t} \wedge D G \circ D_{Y} \Phi_{1}^{t} \circ \sigma^{\prime} \\
& =D G \circ \partial_{\tau} \Phi_{1}^{t} \wedge \partial_{\sigma} \Phi_{1}^{t}+\partial_{\tau} \Phi_{1}^{t} \wedge D G \circ \partial_{\sigma} \Phi_{1}^{t}=\operatorname{div}(G)\left(\partial_{\tau} \Phi_{1}^{t} \wedge \partial_{\sigma} \Phi_{1}^{t}\right) .
\end{aligned}
$$

We compute now directly the value of $J_{1}(t ; t, \sigma)$. We define

$$
T(h)=\frac{\Phi_{1}^{t}(t ; t+h, \sigma)-\Phi_{1}^{t}(t ; t, \sigma)}{h}
$$

and now notice that we can write

$$
\begin{aligned}
\Phi_{1}^{t}(t ; t, \sigma) & =\Phi_{1}^{t}\left(t ; t+h, \Phi_{1}^{t}(t+h ; t, \sigma)\right) \\
& =\Phi_{1}^{t}(t ; t+h, \sigma)+D_{Y} \Phi_{1}^{t}(t ; t+h, \sigma)\left(\Phi_{1}^{t}(t+h ; t, \sigma)-\Phi_{1}^{t}(t ; t, \sigma)\right)+o(h) \\
& =\Phi_{1}^{t}(t ; t+h, \sigma)+h D_{Y} \Phi_{1}^{t}(t ; t+h, \sigma) \circ G(t, \sigma)+o(h) .
\end{aligned}
$$

Now when $h$ goes to zero $D_{Y} \Phi_{1}^{t}(t ; t+h, \sigma) \rightarrow D_{Y} \Phi_{1}^{t}(t ; t, \sigma)=I d$ since $\Phi_{1}^{t}(t ; t, Y)=Y$. Finally, we have $T(h) \rightarrow-G(t, \sigma)$, thus $\partial_{\tau} \Phi_{1}^{t}(t ; t, \sigma)=-G(t, \sigma)$ and $\partial_{\tau} \Phi_{1}^{t} \wedge \partial_{\sigma} \Phi_{1}^{t}(t ; t, \sigma)=-G(t, \sigma) \wedge \sigma^{\prime}=G(t, \sigma) \cdot \nu(\sigma)$. Solving the differential equation between times $\tau$ and $t$ and taking the absolute value then gives the formula (2.5).

- Globally, $\Phi_{1}^{t}$ is bilipschitz. It is possible to show that $\left\|\mid D \Phi_{1}^{t}\right\|_{L^{\infty}([0, t] \times \partial \Omega)} \leq \mathrm{e}^{t\left|\|D G \mid\|_{L^{\infty}([0, T] \times \bar{\Omega}}\right.}$. Using the formula $\left(D \Phi_{1}^{t}\right)^{-1}=J_{1}^{-1}{ }^{t} \operatorname{Com}\left(D \Phi_{1}^{t}\right)$ and the fact that from $(2.5) J_{1}^{-1}$ is bounded on $\bar{\Omega}_{1}^{t}$ thanks to the assumption (2.1) we have \|\|$\left(D \Phi_{1}^{t}\right)^{-1} \|_{L^{\infty}\left(\bar{\Omega}_{1}^{t}\right)}<\infty$. Thus $\Phi_{1}^{t}$ and $\left(\Phi_{1}^{t}\right)^{-1}$ are Lipschitz on $[0, t] \times \partial \Omega \backslash \chi$ and 
$\Omega_{1}^{t} \backslash \chi_{t}$ respectively, and they are both globally continuous on $[0, t] \times \partial \Omega$ and $\bar{\Omega}_{1}^{t}$. Hence they are globally Lipschitz.

Remark A.1. Using the same technique than in the previous proof, we can calculate the derivative of $\Phi_{1}(t ; \tau, \sigma)$ in the $\tau$ direction. Indeed we compute, for all $t, \tau, \sigma$

$$
\begin{aligned}
\Phi_{1}(t ; \tau, \sigma) & =\Phi_{1}\left(t ; \tau+h, \Phi_{1}(\tau+h ; \tau, \sigma)\right) \\
& =\Phi_{1}(t ; \tau+h, \sigma)+D_{Y} \Phi_{1}(t ; \tau+h, \sigma)\left(\Phi_{1}(\tau+h ; \tau, \sigma)-\Phi_{1}(\tau ; \tau, \sigma)\right)+o(h) \\
& =\Phi_{1}(t ; \tau+h, \sigma)+h D_{Y} \Phi_{1}(t ; \tau+h, \sigma) \circ G(\tau, \sigma)+o(h)
\end{aligned}
$$

which gives

$$
\partial_{\tau} \Phi_{1}(t ; \tau, \sigma)=\lim _{h \rightarrow 0} \frac{\Phi_{1}(t ; \tau+h, \sigma)-\Phi_{1}(t ; \tau, \sigma)}{h}=-D_{Y} \Phi_{1}(t ; \tau, \sigma) \circ G(\tau, \sigma) .
$$

\section{REFERENCES}

[1] O. Angulo and J.C. Lopez-Marcos, Numerical schemes for size-structured population equations. Math. Biosci. 157 (1999) 169-188.

[2] D. Barbolosi, A. Benabdallah, F. Hubert and F. Verga, Mathematical and numerical analysis for a model of growing metastatic tumours. Math. Biosci. 218 (2009) 1-14.

[3] D. Barbolosi, C. Faivre and S. Benzekry, Mathematical modeling of MTD and metronomic temozolomide, 2nd Workshop on Metronomic Anti-Angiogenic Chemotherapy in Paediatric Oncology (2010).

[4] C. Bardos, Problèmes aux limites pour les équations aux dérivées partielles du premier ordre à coefficients réels; théorèmes d'approximation; application à l'équation de transport. Ann. Sci. Éc. Norm. Supér. 3 (1970) 185-233.

[5] R. Beals and V. Protopopescu, Abstract time-dependent transport equations. J. Math. Anal. Appl. 2 (1987) $370-405$.

[6] D. Barbolosi and A. Iliadis, Optimizing drug regimens in cancer chemotherapy: a simulation study using a PK-PD model. Comput. Biol. Med. 31 (2001) 157-172.

[7] S. Benzekry, Mathematical analysis of a two-dimensional population model of metastatic growth including angiogenesis, $J$. Evol. Equ. 11 (2011) 187-213.

[8] S. Benzekry, Passing to the limit 2D-1D in a model for metastatic growth, to appear in J. Biol. Dyn., doi:10.1080/17513758.2011.568071.

[9] F. Billy, B. Ribba, O. Saut, H. Morre-Trouilhet, T. Colin, D. Bresch, J. Boissel, E. Grenier and J. Flandrois, A pharmacologically based multiscale mathematical model of angiogenesis and its use in investigating the efficacy of a new cancer treatment strategy. J. Theor. Biol. 260 (2009) 545-562.

[10] F. Boyer, Trace theorems and spatial continuity properties for the solutions of the transport equation. Differential Integral Equations 18 (2005) 891-934.

[11] A. Devys, T. Goudon and P. Laffitte, A model describing the growth and the size distribution of multiple metastatic tumours. Discret. Contin. Dyn. Syst. Ser. B 12 (2009) 731-767.

[12] A. d'Onofrio and A. Gandolfi, Tumour eradication by antiangiogenic therapy: analysis and extensions of the model by Hahnfeldt et al. (1999). Math. Biosci. 191 (2004) 159-184.

[13] A. d'Onofrio, U. Ledzewicz, H. Maurer and H. Schättler, On optimal delivery of combination therapy for tumours. Math. Biosci. 222 (2009) 13-26.

[14] M. Doumic, Analysis of a population model structured by the cells molecular content. Math. Model. Nat. Phenom. 2 (2007) $121-152$.

[15] J.M.L Ebos, C.R. Lee, W. Cruz-Munoz, G.A. Bjarnason, J.G. Christensen and R.S. Kerbel, Accelerated metastasis after short-term treatment with a potent inhibitor of tumour angiogenesis. Cancer Cell 15 (2009) 232-239.

[16] J. Folkman, Antiangiogenesis: new concept for therapy of solid tumours. Ann. Surg. 175 (1972)

[17] P. Hahnfeldt, D. Panigraphy, J. Folkman and L. Hlatky, Tumour development under angiogenic signaling: a dynamical theory of tumour growth, treatment, response and postvascular dormancy. Cancer Res. 59 (1999) 4770-4775.

[18] P. Hahnfeldt, J. Folkman and L. Hlatky, Minimizing long-term tumour burden: the logic for metronomic chemotherapeutic dosing and its antiangiogenic basis. J. Theor. Biol. 220 (2003) 545-554.

[19] K. Iwata, K. Kawasaki and N. Shigesada, A dynamical model for the growth and size distribution of multiple metastatic tumours. J. Theor. Biol. 203 (2000) 177-186.

[20] R.K. Jain, Normalizing tumour vasculature with anti-angiogenic therapy: A new paradigm for combination therapy. Nature Med. 7 (2001) 987-989.

[21] F. Lignet, S. Benzekry, F. Billy, B. Cajavec Bernard, O. Saut, M. Tod, P. Girard, G. Freyer, E. Grenier, T. Colin and B. Ribba, Identifying optimal combinations of anti-angiogenesis drugs and chemotherapies using a theoretical model of vascular tumour growth (in preparation). 
[22] M. Paez-Ribes, E. Allen, J. Hudock, T. Takeda, H. Okuyama, F. Vinals, M. Inoue, G. Bergers, D. Hanahan and O. Casanovas, Antiangiogenic therapy elicits malignant progression of tumours to increased local invasion and distant metastasis. Cancer Cell 15 (2009) 220-231.

[23] B. Perthame, Transport equations in biology. Frontiers in Mathematics, Birkhaüser Verlag, Basel (2007).

[24] G.J. Riely et al., Randomized phase II study of pulse erlotinib before or after carboplatin and paclitaxel in current or former smokers with advanced non-small-cell lung cancer. J. Clin. Oncol. (2009) 264-270.

[25] G.W. Swan, Applications of optimal control theory in biomedicine. Math. Biosci. 101 (1990) $237-284$.

[26] S.L. Tucker and S.O. Zimmerman, A nonlinear model of population dynamics containing an arbitrary number of continuous structure variables. SIAM J. Appl. Math. 48 (1988) 549-591.

[27] B. You, C. Meille, D. Barbolosi, B. tranchand, J. Guitton, C. Rioufol, A. Iliadis and G. Freyer, A mechanistic model predicting hematopoiesis and tumour growth to optimize docetaxel + epirubicin (ET) administration in metastatic breast cancer (MBC): Phase I trial. J. Clin. Oncol.(Meeting abstracts) 25 (2007) 13013. 